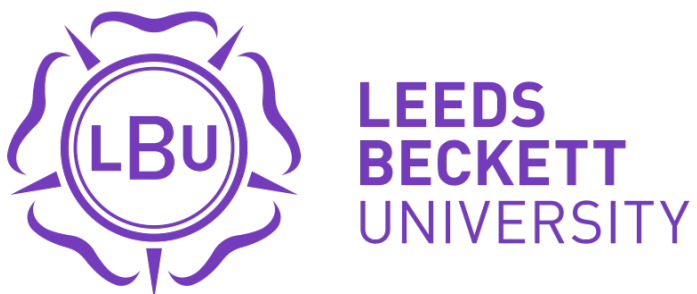

Citation:

Canh, NP and Thanh, SD and Nasir, MA (2020) Nexus between financial development \& energy intensity : Two sides of a coin? Journal of Environmental Management, 270. p. 110902. ISSN 0301-4797 DOI: https://doi.org/10.1016/j.jenvman.2020.110902

Link to Leeds Beckett Repository record:

https://eprints.leedsbeckett.ac.uk/id/eprint/6840/

Document Version:

Article (Accepted Version)

The aim of the Leeds Beckett Repository is to provide open access to our research, as required by funder policies and permitted by publishers and copyright law.

The Leeds Beckett repository holds a wide range of publications, each of which has been checked for copyright and the relevant embargo period has been applied by the Research Services team.

We operate on a standard take-down policy. If you are the author or publisher of an output and you would like it removed from the repository, please contact us and we will investigate on a case-by-case basis.

Each thesis in the repository has been cleared where necessary by the author for third party copyright. If you would like a thesis to be removed from the repository or believe there is an issue with copyright, please contact us on openaccess@leedsbeckett.ac.uk and we will investigate on a case-by-case basis. 


\title{
Nexus between Financial Development \& Energy Intensity: two sides of a coin?
}

\author{
Nguyen Phuc Canh* \\ School of Banking, University of Economics Ho Chi Minh City, Vietnam \\ canhnguyen@ueh.edu.vn \\ Su Dinh Thanh \\ School of Public Finance, University of Economics Ho Chi Minh City, Vietnam \\ dinhthanh@ueh.edu.vn \\ Muhammad Ali Nasir \\ Leeds Business School, Leeds Beckett University, Leeds LS1 3HB, United Kingdom \\ M.A.Nasir@leedsbeckett.ac.uk
}

\begin{abstract}
This study examines the multidimensional impact of financial development (FD) on consumption energy intensity and production energy intensity. A global sample of 81 economies consisting of three subsamples (29 high income [HIEs], 21 upper middle income [UMEs] and 31 low and lower middle income [LMEs]) from 1997 to 2013 is analyzed by employing several estimators and an inclusive estimation strategy for empirical robustness. Our key findings suggest that: First, FD is broadly found to increase production energy intensity except for the negative effects of financial institutions' efficiency. The results also confirm the long-run relationship. The financial depth and financial access appear to reduce consumption energy intensity, while financial efficiency has the opposite effect. Financial institutions have increasing, while financial markets have decreasing impact on consumption energy intensity in the long run. Second, in the face of an oil price shock, countries with higher levels of FD experience a reduce production energy intensity, while the countries with stronger financial institutions experience a reduction in consumption energy intensity, however, opposite is true in the case of financial markets. Third, the FD appears to decrease production energy intensity in HIEs, while it has increasing effect in UMEs and mixed effects in LMEs. Meanwhile, the FD induces higher consumption energy intensity in LMEs, which is same for the impacts of financial markets in UMEs and HIEs. Lastly, financial institutions appear to reduce consumption energy intensity in UMEs and HIEs. Our findings have profound policy implications in the context of the debate on the role of finance in energy efficiency.
\end{abstract}

Keywords: Energy consumption; efficiency; production; financial institutions; financial markets; panel data.

JEL codes: E44, Q41, Q43. 


\section{Introduction}

In the context of efforts to tackle climate change, the improvement in the efficiency of energy usage is emphasized as one of the most important goals for sustainable development ( $\underline{\mathrm{UN}}$, 2019). Theoretically, the determinants of energy consumption are broken down to the Influence, Population, Affluence, and Technology factors in IPAT model by Ehrlich and Holdren (1971) and the STIRPAT model by Dietz and Rosa (1997). Empirically, economic development, urbanization, industrialization, or economic openness are often found to have a significant impact on energy consumption (Hanif, 2018), though, the results are contrasting, owing to the fact that these factors and their impact require to be seen in a context and can be influenced by catalysing and moderating factors. Concomitantly, this study is carried out by the following motivations and gaps in the existing body of knowledge. First, the energy usage for consumption or production is different in purpose. While the energy usage for consumption is dependent on lifestyle (urbanisation and affluence), the energy usage for production would be associated with the level of technological progress. However, most of the studies in existing literature have not accounted for this demarcation in their empirical investigations (e.g., see Gaies et al. (2019)). Second, the literature on the impact of financial development (FD) on energy consumption suggests mixed results which require further exploration of moderating factors. FD is documented as an important driver of higher energy consumption (Alam et al., 2015), but it is also noticed to have benefits for the environment through support for renewable energy and higher energy efficiency ( $\underline{\mathrm{Al}}$ Mamun et al., 2018). The financial sector and markets are arguably the component of the overall institutional framework (Blackburn et al., 2012), which provides financial resources (or financial access) for economic activities (Berdiev and Saunoris, 2016). Therefore, a higher FD could lead to higher energy consumption and energy intensity (see Adams and Klobodu (2018) among several empirical studies). However, a higher FD, especially financial efficiency, can also provide funds and financial services with lower costs (Svirydzenka, 2016), creating favourable conditions for technological advancement and economic transformation toward higher energy efficiency. Third, several previous studies employed simple proxies of FD such as credit or stock market capitalization (e.g., see Maskus et al. (2019)). These proxies mostly represent a single aspect of financialization e.g. financial 
institutions depth only (Svirydzenka, 2016), while it is vital that the FD is considered in a broader context by including the main components i.e. financial institutions and financial markets, and dimensions i.e. financial depth, financial access, and financial efficiency (Svirydzenka, 2016). In this context, empirical evidence shows that these components and dimensions of the financial sector may have different impacts on economic activities ( $\underline{\text { Botev }}$ et al., 2019). It is intriguing to investigate the impact of different components and dimensions of financial development on energy consumption to gain a deeper insight into the nexus between them. Notably, Coban and Topcu (2013) emphasize that the impacts of FD on energy consumption depending on the measurement of FD. Forth, energy consumption is a function of demand and price, whereas the associations between FD and energy prices on energy intensity is underexplored in the existing literature. One can expect that the increase in energy price would induce higher cost of energy consumption. Common economic wisdom would suggest a decrease in energy consumption or a transformation in energy consumption toward higher efficiency in a condition of higher energy price. However, the transformation of energy consumption, especially in technology, needs huge investment, which is, in return, out of economic agencies' interests (Geng et al., 2016). Thus, a higher FD may play important roles in helping a country to transform its energy consumption while facing external cost shocks in the form of higher energy prices. Yet, this phenomenon needs further exploration and robust empirical testing.

This study at first attempts to extend the literature on the determinants of FD on energy intensity from a multidimensional perspective. Specifically, we examine the impacts of three financial dimensions, financial depth, financial access, and financial efficiency of both financial institutions and financial markets on energy intensity in a global sample. Furthermore, the study analyzes the impacts of FD on energy intensity by both aspects of production and consumption. Consequently, we also examine the roles of FD in helping a country to transform its energy consumption toward higher efficiency under the cost shocks in the form of higher energy prices. Lastly, the impact of FD on energy intensity is carefully examined for three sub-income groups. Empirically, a sample of 81 economies and three subsamples including 29 HIEs, 21 UMEs, and 31 LMEs are examined by employing several econometrical approaches to panel data. The two-step system GMM estimator is applied to 
deal with endogeneity, heteroscedasticity, and autoregression, while feasible generalized least square (FGLS) and panel corrected standard errors (PCSE) are also employed for robustness testing. To examine the impacts of FD on energy intensity, in the long run, the canonical correlation regression (CCR) model is used as the main estimator, while panel fully modified OLS (FMOLS) estimator is employed for robustness check. Notably, we estimate the impacts of the interaction term between FD and oil price and perform predictive margins analysis for them to investigate the roles of FD in transforming the energy intensity of countries facing energy price shocks.

This study contributes to the literature in four dimensions. First, the study examines the determinants of energy intensity in two aspects: consumption energy intensity and production energy intensity. The analysis for both aspects of consumption and production is essential to understand the dynamics of energy consumption and draw policy implications for higher energy efficiency. Second, the study is the first attempt in examining multiple dimensions of financial development including financial depth, financial access, and financial efficiency in both financial institutions and financial markets and their impact on energy intensity. This helps to fulfil the research gap in the existing body of knowledge on the relationship between financial development and energy consumption. Third, the study analyzes the roles of financial development in transforming the energy consumption toward efficiency in the context of high energy price. This highlights the important catalyst role of financial development in energy transformation. Lastly, the study provides empirical evidence on these aspects in a global sample and time horizon along with the analysis of three subsamples by income level, following the income classification of the World Bank.

The rest of the paper is structured as follows. Next section briefly and critically reviews the existing literature on the subject. The methodology, data, and estimation strategy are explained in Section 3. Empirical results are presented in Section 4. The final Section 5 entails discussions and conclusion.

\section{Literature review}

In the recent literature on the subject, attention has been paid to the role of FD in tackling environmental change and more specifically its role in the dynamics of energy consumption. 
For instance, Farhani and Ozturk (2015) find the causal relationships between economic development, FD, CO2 emissions, energy consumption in Tunisia. Al-Mulali et al. (2015) document the positive impacts of GDP growth, urbanization, and $\mathrm{FD}$ on $\mathrm{CO}_{2}$ emissions in 23 selected European countries. Shahbaz et al. (2020) find the increasing effect of FD on $\mathrm{CO}_{2}$ emissions in the case of the United Arab Emirates. Le and Ozturk (2020) demonstrate that globalization, FD, and energy consumption are the main causes of the increases in $\mathrm{CO} 2$ emissions for a sample of 47 Emerging Market and Developing Economies. Recently, Le et al. (2020) emphasize that the development of the financial sector is a significant driver of renewable energy consumption in a sample of 55 countries. The review of the literature on the subject suggests that little attention has been paid to the impact of FD on energy intensity.

On one hand, the FD is an important component of the overall institutional framework (Blackburn et al., 2012), which helps to provide financial resources (or financial access) for

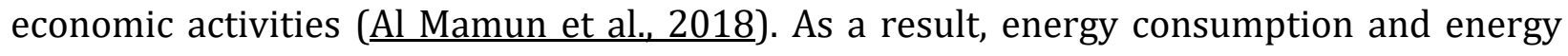
intensity could be exacerbated due to higher consumption, investment, and production. Several studies show a positive impact of FD on energy consumption (e.g., see Adams and Klobodu (2018) on 26 African countries (1985-2011) among others). On the other hand, the FD is argued to play an important role in technological progress and energy consumption transition. For instance, some studies show the important role of FD in energy transformation toward renewable energies (e.g., see Ali et al. (2018) for the case of 19 Asia Cooperation Dialogue member countries). A few studies also focus on FD roles in energy efficiency. Chen et al. (2019) reveal that FD exerts a significant negative effect on energy intensity for non-OECD countries, but it has a limited impact on energy reduction for OECD countries. Pan et al. (2019a) indicate that FD had a causal impact on energy intensity in Bangladesh, but it is reduced latterly. Yet, Pan et al. (2019b) further add that FD has some roles to influence energy intensity in Bangladesh.

The financial sector has two main sub-sectors or components which are financial institutions (i.e. banks, insurance companies, funds, venture capital firms, and other types of non-bank financial institutions) and financial markets (i.e. stock markets, bond markets, wholesale money markets, and by-passing traditional bank lending), both have different roles in 
economic activities (Svirydzenka, 2016). The financial institutions would be more important channels of finance to broaden the range of economic agencies including firm and citizens, while financial markets are likely to be supportive toward investment activities of firms and institutional organizations (Svirydzenka, 2016). This implies that financial institutions and financial markets may have a heterogeneous impact on energy intensity. However, most of the previous stud have been using simple proxies to measure FD. Maskus et al. (2019), for instance, employ private credit and stock market capitalization. Some other indicators are utilized such as money supply (Cavalcante et al., 2018), credit to the private sector ( et al., 2018) or stock traded and stock market capitalization (Yang, 2019). However, it is vital to have an inclusive measure of financial development which can encompass the various aspect of financialization. Some recent studies, for instance, Nasir et al. (2019) on ASEAN countries tried to include more than one measure of financial development. In this context, Svirydzenka (2016) emphasize that there are three dimensions of each component or subsector of financial sector including financial depth (i.e., size and liquidity of markets), financial access (i.e. the ability of individuals and companies to access financial services), and financial efficiency (i.e. the ability of institutions to provide financial services at low cost and with sustainable revenues, and the level of activity of capital markets). That is, the financial depth and financial access may have inducing effects on energy intensity as it reduces financial constraints and provides fund for most of the economic agencies. In contrast, the financial efficiency may play as a good factor toward higher energy efficiency since it provides finance with lower costs, which is a positive factor for technological progress and energy transformation, which are impacted by the large cost of transformation.

In addition to employing an inclusive measure of financial developed, it is also vital to account for the catalysing factor. Interestingly, Bhattacharyya and Hodler (2014) suggest that the studies in FD should use additional control variables as well as different samples, and alternative measures of FD. In this regard, the energy price is one of an important driver of energy usage since it impacts on the cost of energy. Logically, the higher energy price would lead to higher cost of production and also consumption that can lead to the drop in energy demand. On the other hand, the higher costs can stimulate the changes in energy usage for production and consumption toward higher efficiency. However, the literature 
shows that the energy transformation toward efficiency or greener sources faces financial constraints (Ii and Zhang, 2019). In this situation, the higher FD may play as a good catalyst for energy transformation toward higher efficiency. Concomitantly, the subject study intends to analyses the multidimensional role of FD in consumption energy intensity and production energy intensity and moderating role of energy prices and in the next section, we elaborate the empirical approach to facilitate this endeavour.

\section{Methodology and data}

On the theoretical grounds, we can track the roots of our empirical approach to the model of human impact on the environment (I) as the product of three factors: population (P), affluence (A) and technology (T) (namely IPAT model) based on the seminal work by Ehrlich and Holdren (1971) and the stochastic impacts by regression on population, affluence and technology (namely STIRPAT model) by Dietz and Rosa (1997) to specify the energy intensity function with common drivers including economic development (Income), industrialization (Industry), urbanization (Urban). These drivers have been used in several previous empirical studies ( added as the price determinant from the demand side (Omri and Nguyen, 2014), while trade openness (Trade) and FDI inflows (FDI) are added as the proxies of economic integration (Phuc Nguyen et al., 2019). Moreover, the energy supply (ES) is included to proxy for the supply side's determinant (Azam et al., 2015). As the fact of the transformation in energy use is a long-term process (Pradhan et al., 2018), the empirical investigation of energy intensity is formed by dynamic panel estimation:

$$
\begin{gathered}
E I_{i t}=\alpha_{0}+\alpha_{1} E I_{i t-1}+\beta_{1} \text { Income }_{i t}+\beta_{2} \text { Industry }_{i t}+\beta_{3} \text { Urban }_{i t}+\beta_{4} \text { Trade }_{i t}+\beta_{5} F D I_{i t} \\
+\beta_{6} O P_{i t}+\beta_{7} E S_{i t}+\beta_{8} \text { FIN }_{i t}+\gamma_{i}+\varepsilon_{i t}
\end{gathered}
$$

in which: $i, t$ denote for country $i$ at year $t . \alpha$ and $\beta$ are estimated coefficients. $\gamma_{i}$ is country effects and $\varepsilon_{i t}$ is residual terms.

In term of energy intensity, it is hard to divide the energy intensity in production or consumption due to the complication in the usage of energy. There is no separated data on 
energy use for production or consumption. The data from the International Energy Association (IEA, US) provides us with total primary energy consumption. Taking advantage of this database, this study tries to extend the literature of energy consumption to two main aspects: production energy intensity $(P E I)$ and consumption energy intensity (CEI), separately. First, the primary energy consumption as a ratio of GDP is collected from World Development Indicators (WDIs - World Bank) and taking logarithms to proxy for production energy intensity. This indicator implies the efficiency in using energy to produce one unit of output. Second, the total primary energy consumption is collected from EIA and divided for the total population (from WDIs) to form total primary energy consumption per capita. This indicator is taken in logarithms and implies energy consumption by one citizen. Since we cannot completely divide energy use for production or consumption thus there can be some limitations for using these proxies, but they are likely to be the most reliable separate indicators of efficiency in energy use for production and consumption.

The energy intensity in production is strongly linked to the production structures, whereas the industrialization would be one of the important drivers (Lin and Zhu, 2017). The energy intensity in citizens consumption is related to the lifestyle and thus the urbanization is a very important factor (Zhang et al., 2017). Therefore, the study estimates the influences of FD on energy intensity in production and consumption, separately, with some minor adjustments. The industrialization is kept as an important determinant of production energy intensity ( $E q$. [2a]), while the urbanization is kept as an important driver of consumption energy intensity (Eq. [2b]) as follows:

$$
\begin{aligned}
\text { PEI }_{i t}=\alpha_{0}+ & \alpha_{1} P E I_{i t-1}+\beta_{1} \text { Income }_{i t}+\beta_{2} \text { Industry }_{i t}+\beta_{3} \text { Trade }_{i t}+\beta_{4} F D I_{i t}+\beta_{5} O P_{i t} \\
& +\beta_{6} E_{i t}+\beta_{7} \text { FIN }_{i t}+\gamma_{i}+\varepsilon_{i t} \quad[2 \mathrm{a}] \\
C E I_{i t}=\alpha_{0}+ & \alpha_{1} C E I_{i t-1}+\beta_{1} \text { Income }_{i t}+\beta_{2}{ }_{2} \text { Urban }_{i t}+\beta_{3} \text { Trade }_{i t}+\beta_{4} F D I_{i t}+\beta_{5} O P_{i t} \\
& +\beta_{6} E S_{i t}+\beta_{7} \text { FIN }_{i t}+\gamma_{i}+\varepsilon_{i t} \quad[2 \mathrm{~b}]
\end{aligned}
$$

In term of control variables, the logarithm of real GDP per capita, industrial value added (\% GDP), urban population (\% total population), trade openness (\% GDP), FDI net inflows (\% GDP) are collected from WDIs to proxy for economic development level, industrialization, urbanization, trade openness, and FDI, respectively, which are used in several previous 
studies (e.g., see Phuc Nguyen et al. (2019), Canh et al. (2019), Nguyen et al. (2018) among others). The annual average crude oil price (West Texas Intermediate - Cushing, Oklahoma) is collected from the Federal Reserve Economic Data (Fred, St. Louis Fed US) and taking logarithms to proxy for energy price. At last, total primary energy production is divided for the total population (from WDIs) and taking logarithms to proxy for energy supply. In term of FD, the study collects nine indicators from Financial development database of IMF (FDIMF) including overall financial development $(O F D)$, two overall indicators of two subsectors as financial institutions $(F I)$ and financial markets $(F M)$, three dimensions of each sub-sector as financial institutions depth (FID), financial institutions access (FIA), and financial institutions efficiency ( $F I E$ ) of financial institutions and financial markets depth $(F M D)$, financial markets access $(F M A)$, and financial markets efficiency $(F M E)$ of financial markets.

As the availability of primary energy intensity per GDP from WDIs to 2013 (see column 5 in Table 1 for the data range), while most of the countries have data on primary energy consumption from the late of the 1990s, the final sample includes 81 countries over the period 1997-2013 as the best sample with largest countries and longest time period (see table A1, Appendix, for the list of countries). Table 1 presents detail on variables, definitions, measurements, sources, and data description. Besides the full global sample of 81 economies, this study uses the income classification from World Bank to divide the sample into three subsamples including 29 high income (HIEs), 21 upper middle income (UMEs) and 31 low and lower middle income (LMEs). The analysis for subsamples by income level would be helpful in identifying the different impacts of FD on the energy intensity by income level (Sadorsky, 2013). The data description for three subsamples is reported in Table A2, Appendix. 
Table 1. Variables, definitions, measurements, sources, and data description

\begin{tabular}{|c|c|c|c|c|c|c|c|c|c|}
\hline Variable & Definitions & Measurements & Sources & Data range & Obs & Mean & SD. & Min & Max \\
\hline PEI & $\begin{array}{l}\text { Production Energy } \\
\text { intensity }\end{array}$ & $\begin{array}{l}\text { Log of Energy intensity level of primary energy } \\
\text { (MJ/\$2011 PPP GDP) }\end{array}$ & WDIs & $1990-2015$ & 1,377 & 1.70 & 0.47 & 0.65 & 3.62 \\
\hline CEI & $\begin{array}{l}\text { Consumption Energy } \\
\text { intensity }\end{array}$ & $\begin{array}{l}\text { Log of Primary energy consumption per capita } \\
\text { (kg/person)a }\end{array}$ & WDIs \& EIA & $1980-2013$ & 1,377 & 6.71 & 1.55 & 3.07 & 9.09 \\
\hline Income & $\begin{array}{l}\text { Economic } \\
\text { development }\end{array}$ & Log of GDP per capita (constant 2010 US $\$$ ) & WDIs & $1960-2018$ & 1,377 & 8.42 & 1.53 & 5.42 & 11.24 \\
\hline Industry & Industrialization & $\begin{array}{l}\text { Industry (including construction), value added (\% of } \\
\text { GDP) }\end{array}$ & WDIs & $1960-2018$ & 1,377 & 25.75 & 7.11 & 11.26 & 48.53 \\
\hline Urban & Urbanization & Urban population (\% of total population) & WDIs & $1960-2018$ & 1,377 & 55.18 & 22.04 & 11.83 & 94.84 \\
\hline Trade & Trade openness & Trade (\% of GDP) & WDIs & $1960-2018$ & 1,377 & 76.65 & 35.67 & 16.44 & 220.4 \\
\hline FDI & FDI inflows & Foreign direct investment, net inflows (\% of GDP) & WDIs & $1960-2018$ & 1,377 & 3.98 & 4.66 & -15.99 & 50.50 \\
\hline $\mathrm{OP}$ & Energy price & $\begin{array}{l}\text { Log of Crude Oil Prices (West Texas Intermediate - } \\
\text { Cushing, Oklahoma, Dollars per Barrel, average } \\
\text { annual) }\end{array}$ & Fred & $1986-2019$ & 1,377 & 3.92 & 0.61 & 2.67 & 4.60 \\
\hline ES & $\begin{array}{l}\text { Primary Energy } \\
\text { supply }\end{array}$ & $\begin{array}{l}\text { Log of Primary Energy production per capita } \\
\text { (kg/person) }\end{array}$ & $\begin{array}{l}\text { WDIs and } \\
\text { EIA }\end{array}$ & $1980-2013$ & 1,377 & 5.51 & 2.20 & -0.46 & 9.60 \\
\hline OFD & $\begin{array}{l}\text { Overall financial } \\
\text { development }\end{array}$ & Overall financial development index & FD-IMF & $1986-2017$ & 1,377 & 0.35 & 0.24 & 0.04 & 1.00 \\
\hline FI & $\begin{array}{l}\text { Financial institutions } \\
\text { development }\end{array}$ & Financial institutions index & FD-IMF & $1986-2017$ & 1,377 & 0.44 & 0.23 & 0.08 & 1.00 \\
\hline FM & $\begin{array}{l}\text { Financial markets } \\
\text { development }\end{array}$ & Financial markets index & FD-IMF & $1986-2017$ & 1,377 & 0.26 & 0.26 & 0.00 & 1.00 \\
\hline FID & $\begin{array}{l}\text { Financial institutions } \\
\text { depth }\end{array}$ & Financial institutions depth index & FD-IMF & $1986-2017$ & 1,377 & 0.30 & 0.28 & 0.01 & 1.00 \\
\hline FIA & $\begin{array}{l}\text { Financial institutions } \\
\text { Access }\end{array}$ & Financial institutions Access index & FD-IMF & $1986-2017$ & 1,377 & 0.34 & 0.29 & 0.00 & 1.00 \\
\hline FIE & $\begin{array}{l}\text { Financial institutions } \\
\text { Efficiency }\end{array}$ & Financial institutions efficiency index & FD-IMF & $1986-2017$ & 1,377 & 0.63 & 0.16 & 0.11 & 0.94 \\
\hline FMD & $\begin{array}{l}\text { Financial markets } \\
\text { depth }\end{array}$ & Financial markets depth index & FD-IMF & $1986-2017$ & 1,377 & 0.24 & 0.28 & 0.00 & 1.00 \\
\hline FMA & $\begin{array}{l}\text { Financial markets } \\
\text { Access }\end{array}$ & Financial markets Access index & FD-IMF & $1986-2017$ & 1,377 & 0.24 & 0.28 & 0.00 & 1.00 \\
\hline FME & $\begin{array}{l}\text { Financial markets } \\
\text { Efficiency }\end{array}$ & Financial markets efficiency index & FD-IMF & $1986-2017$ & 1,377 & 0.29 & 0.35 & 0.00 & 1.00 \\
\hline
\end{tabular}

Note: a: the primary energy consumption per capita is calculated by dividing the total primary energy consumption (from EIA) for total population

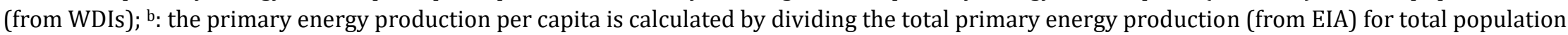
(from WDIs). WDIs is World Development Indicators database (World Bank, 2020); EIA is international data on energy of U.S. Energy Information Administration; Fred is the Federal Reserve Economic Data (FRED) of St. Louis Fed, US; FD-IMF is financial development database of IMF. 
Table 2. Unconditional correlation matrix

\begin{tabular}{|c|c|c|c|c|c|c|c|c|c|c|c|c|c|c|c|c|c|c|}
\hline Correlation & PEI & CEI & Income & Industry & Urban & Trade & FDI & OP & ES & OFD & FI & FM & FID & FIA & FIE & FMD & FMA & FME \\
\hline PEI & 1.00 & & & & & & & & & & & & & & & & & \\
\hline CEI p-value & $\begin{array}{l}-0.17 \\
0.00\end{array}$ & 1.00 & & & & & & & & & & & & & & & & \\
\hline $\begin{array}{l}\text { Income } \\
\quad p \text {-value }\end{array}$ & $\begin{array}{l}-0.40 \\
0.00\end{array}$ & $\begin{array}{l}0.90 \\
0.00\end{array}$ & 1.00 & & & & & & & & & & & & & & & \\
\hline $\begin{array}{c}\text { Industry } \\
p \text {-value }\end{array}$ & $\begin{array}{l}-0.08 \\
0.00\end{array}$ & $\begin{array}{l}0.32 \\
0.00\end{array}$ & $\begin{array}{l}0.19 \\
0.00\end{array}$ & 1.00 & & & & & & & & & & & & & & \\
\hline $\begin{array}{l}\text { Urban } \\
p \text {-value }\end{array}$ & $\begin{array}{l}-0.29 \\
0.00\end{array}$ & $\begin{array}{l}0.81 \\
0.00\end{array}$ & $\begin{array}{l}0.81 \\
0.00\end{array}$ & $\begin{array}{l}0.22 \\
0.00\end{array}$ & 1.00 & & & & & & & & & & & & & \\
\hline $\begin{array}{l}\text { Trade } \\
p \text {-value }\end{array}$ & $\begin{array}{l}-0.03 \\
0.23\end{array}$ & $\begin{array}{l}0.28 \\
0.00\end{array}$ & $\begin{array}{l}0.18 \\
0.00\end{array}$ & $\begin{array}{l}0.28 \\
0.00\end{array}$ & $\begin{array}{l}0.10 \\
0.00\end{array}$ & 1.00 & & & & & & & & & & & & \\
\hline FDI $\quad p$-value & $\begin{array}{l}-0.02 \\
0.43\end{array}$ & $\begin{array}{l}0.09 \\
0.00\end{array}$ & $\begin{array}{l}0.07 \\
0.01\end{array}$ & $\begin{array}{l}0.01 \\
0.62\end{array}$ & $\begin{array}{l}0.07 \\
0.01\end{array}$ & $\begin{array}{l}0.39 \\
0.00\end{array}$ & 1.00 & & & & & & & & & & & \\
\hline $\mathrm{OP} \quad p$-value & $\begin{array}{l}-0.20 \\
0.00\end{array}$ & $\begin{array}{l}0.03 \\
0.21\end{array}$ & $\begin{array}{l}0.09 \\
0.00\end{array}$ & $\begin{array}{l}-0.01 \\
0.84\end{array}$ & $\begin{array}{l}0.07 \\
0.01\end{array}$ & $\begin{array}{l}0.14 \\
0.00\end{array}$ & $\begin{array}{l}0.16 \\
0.00\end{array}$ & 1.00 & & & & & & & & & & \\
\hline$p$-value & $\begin{array}{l}-0.11 \\
0.00\end{array}$ & $\begin{array}{l}0.81 \\
0.00\end{array}$ & $\begin{array}{l}0.71 \\
0.00\end{array}$ & $\begin{array}{l}0.40 \\
0.00\end{array}$ & $\begin{array}{l}0.64 \\
0.00\end{array}$ & $\begin{array}{l}0.09 \\
0.00\end{array}$ & $\begin{array}{l}0.00 \\
1.00\end{array}$ & $\begin{array}{l}0.05 \\
0.06\end{array}$ & 1.00 & & & & & & & & & \\
\hline OFD $p$-value & $\begin{array}{l}-0.27 \\
0.00\end{array}$ & $\begin{array}{l}0.74 \\
0.00\end{array}$ & $\begin{array}{l}0.84 \\
0.00\end{array}$ & $\begin{array}{l}0.14 \\
0.00\end{array}$ & $\begin{array}{l}0.61 \\
0.00\end{array}$ & $\begin{array}{l}0.07 \\
0.01\end{array}$ & $\begin{array}{l}0.03 \\
0.30\end{array}$ & $\begin{array}{l}0.10 \\
0.00\end{array}$ & $\begin{array}{l}0.63 \\
0.00\end{array}$ & 1.00 & & & & & & & & \\
\hline$p$-value & $\begin{array}{l}-0.31 \\
0.00\end{array}$ & $\begin{array}{l}0.76 \\
0.00\end{array}$ & $\begin{array}{l}0.88 \\
0.00\end{array}$ & $\begin{array}{l}0.07 \\
0.01\end{array}$ & $\begin{array}{l}0.62 \\
0.00\end{array}$ & $\begin{array}{l}0.16 \\
0.00\end{array}$ & $\begin{array}{l}0.07 \\
0.01\end{array}$ & $\begin{array}{l}0.16 \\
0.00\end{array}$ & $\begin{array}{l}0.61 \\
0.00\end{array}$ & $\begin{array}{l}0.94 \\
0.00\end{array}$ & 1.00 & & & & & & & \\
\hline$p$-value & $\begin{array}{l}-0.21 \\
0.00\end{array}$ & $\begin{array}{l}0.65 \\
0.00\end{array}$ & $\begin{array}{l}0.73 \\
0.00\end{array}$ & $\begin{array}{l}0.19 \\
0.00\end{array}$ & $\begin{array}{l}0.53 \\
0.00\end{array}$ & $\begin{array}{l}-0.02 \\
0.54\end{array}$ & $\begin{array}{l}-0.01 \\
0.75\end{array}$ & $\begin{array}{l}0.05 \\
0.07\end{array}$ & $\begin{array}{l}0.59 \\
0.00\end{array}$ & $\begin{array}{l}0.96 \\
0.00\end{array}$ & $\begin{array}{l}0.80 \\
0.00 \\
\end{array}$ & 1.00 & & & & & & \\
\hline FID $p$-value & $\begin{array}{l}-0.27 \\
0.00\end{array}$ & $\begin{array}{l}0.66 \\
0.00\end{array}$ & $\begin{array}{l}0.79 \\
0.00\end{array}$ & $\begin{array}{l}0.07 \\
0.01\end{array}$ & $\begin{array}{l}0.54 \\
0.00\end{array}$ & $\begin{array}{l}0.13 \\
0.00\end{array}$ & $\begin{array}{l}0.06 \\
0.03\end{array}$ & $\begin{array}{l}0.10 \\
0.00\end{array}$ & $\begin{array}{l}0.55 \\
0.00\end{array}$ & $\begin{array}{l}0.90 \\
0.00\end{array}$ & $\begin{array}{l}0.91 \\
0.00\end{array}$ & $\begin{array}{l}0.80 \\
0.00\end{array}$ & 1.00 & & & & & \\
\hline FIA $p$-value & $\begin{array}{l}-0.28 \\
0.00\end{array}$ & $\begin{array}{l}0.75 \\
0.00\end{array}$ & $\begin{array}{l}0.84 \\
0.00\end{array}$ & $\begin{array}{l}-0.02 \\
0.42\end{array}$ & $\begin{array}{l}0.00 \\
0.64 \\
0.00\end{array}$ & $\begin{array}{l}0.10 \\
0.00\end{array}$ & $\begin{array}{l}0.06 \\
0.04\end{array}$ & $\begin{array}{l}0.16 \\
0.00\end{array}$ & $\begin{array}{l}0.59 \\
0.00\end{array}$ & $\begin{array}{l}0.83 \\
0.00\end{array}$ & $\begin{array}{l}0.91 \\
0.00 \\
\end{array}$ & $\begin{array}{l}0.68 \\
0.00 \\
\end{array}$ & $\begin{array}{l}0.70 \\
0.00\end{array}$ & 1.00 & & & & \\
\hline FIE $p$-value & $\begin{array}{l}-0.24 \\
0.00\end{array}$ & $\begin{array}{l}0.47 \\
0.00\end{array}$ & $\begin{array}{l}0.54 \\
0.00\end{array}$ & $\begin{array}{l}0.22 \\
0.00\end{array}$ & $\begin{array}{l}0.32 \\
0.00\end{array}$ & $\begin{array}{l}0.26 \\
0.00\end{array}$ & $\begin{array}{l}0.07 \\
0.01\end{array}$ & $\begin{array}{l}0.15 \\
0.00\end{array}$ & $\begin{array}{l}0.37 \\
0.00\end{array}$ & $\begin{array}{l}0.65 \\
0.00\end{array}$ & $\begin{array}{l}0.71 \\
0.00\end{array}$ & $\begin{array}{l}0.53 \\
0.00\end{array}$ & $\begin{array}{l}0.58 \\
0.00\end{array}$ & $\begin{array}{l}0.49 \\
0.00\end{array}$ & 1.00 & & & \\
\hline FMD $p$-value & $\begin{array}{l}-0.19 \\
0.00\end{array}$ & $\begin{array}{l}0.61 \\
0.00\end{array}$ & $\begin{array}{l}0.71 \\
0.00\end{array}$ & $\begin{array}{l}0.11 \\
0.00\end{array}$ & $\begin{array}{l}0.53 \\
0.00\end{array}$ & $\begin{array}{l}0.01 \\
0.60\end{array}$ & $\begin{array}{l}0.01 \\
0.69\end{array}$ & $\begin{array}{l}0.13 \\
0.00\end{array}$ & $\begin{array}{l}0.55 \\
0.00\end{array}$ & $\begin{array}{l}0.92 \\
0.00\end{array}$ & $\begin{array}{l}0.82 \\
0.00\end{array}$ & $\begin{array}{l}0.92 \\
0.00\end{array}$ & $\begin{array}{l}0.87 \\
0.00\end{array}$ & $\begin{array}{l}0.65 \\
0.00\end{array}$ & $\begin{array}{l}0.53 \\
0.00\end{array}$ & 1.00 & & \\
\hline FMA $p$-value & $\begin{array}{l}-0.33 \\
0.00\end{array}$ & $\begin{array}{l}0.60 \\
0.00\end{array}$ & $\begin{array}{l}0.72 \\
0.00\end{array}$ & $\begin{array}{l}0.19 \\
0.00\end{array}$ & $\begin{array}{l}0.53 \\
0.00\end{array}$ & $\begin{array}{l}0.04 \\
0.15\end{array}$ & $\begin{array}{l}0.01 \\
0.70\end{array}$ & $\begin{array}{l}0.04 \\
0.13\end{array}$ & $\begin{array}{l}0.53 \\
0.00\end{array}$ & $\begin{array}{l}0.82 \\
0.00\end{array}$ & $\begin{array}{l}0.72 \\
0.00\end{array}$ & $\begin{array}{l}0.83 \\
0.00\end{array}$ & $\begin{array}{l}0.72 \\
0.00\end{array}$ & $\begin{array}{l}0.63 \\
0.00\end{array}$ & $\begin{array}{l}0.45 \\
0.00\end{array}$ & $\begin{array}{l}0.72 \\
0.00\end{array}$ & 1.00 & \\
\hline FME $p$-value & $\begin{array}{l}-0.04 \\
0.17\end{array}$ & $\begin{array}{l}0.49 \\
0.00\end{array}$ & $\begin{array}{l}0.49 \\
0.00\end{array}$ & $\begin{array}{l}0.19 \\
0.00\end{array}$ & $\begin{array}{l}0.35 \\
0.00\end{array}$ & $\begin{array}{l}-0.09 \\
0.00\end{array}$ & $\begin{array}{l}-0.04 \\
0.13\end{array}$ & $\begin{array}{l}-0.04 \\
0.10\end{array}$ & $\begin{array}{l}0.46 \\
0.00\end{array}$ & $\begin{array}{l}0.74 \\
0.00\end{array}$ & $\begin{array}{l}0.55 \\
0.00\end{array}$ & $\begin{array}{l}0.84 \\
0.00\end{array}$ & $\begin{array}{l}0.50 \\
0.00\end{array}$ & $\begin{array}{l}0.50 \\
0.00\end{array}$ & $\begin{array}{l}0.39 \\
0.00\end{array}$ & $\begin{array}{l}0.68 \\
0.00\end{array}$ & $\begin{array}{l}0.49 \\
0.00\end{array}$ & 1.00 \\
\hline
\end{tabular}


Table 2 presents the unconditional correlation matrix. It shows that production energy intensity has negative correlations between all control and explanatory variables, while consumption energy intensity has positive ones. It may imply a higher energy efficiency of production along with economic development, urbanization, industrialization, economic integration, and financial development. These economic factors are likely to be in line with higher consumption energy intensity as the positive correlations indicate.

In estimating Eq. [2a] and [2b], the endogeneity is a serious issue as the dynamic panel models and the possible existence of feedback effects from energy consumption to other economic factors such as economic development and FD. Moreover, there may be other kinds of endogeneity such as unobserved heterogeneity, simultaneity and dynamic endogeneity (Ullah et al., 2018). In such a case, the traditional fixed effects estimator is inconsistent (Nickell, 1981). The first difference methods by Anderson and Hsiao (1982) is proposed to be used (Hansen, 1982). Later, Arellano and Bond (1991) proposed the GMM estimator as a more efficient estimation approach. However, the method in Arellano-Bond GMM estimator faces the problem of asymptotically and considerable bias in unbalanced panel data (Roodman, 2006). The system GMM estimator proposed by Arellano and Bover (1995) which was extended by Blundell and Bond (1998) reduces the bias associated with the fixed effects in short panels and is named as two-step system GMM (Roodman, 2009). The two-step system GMM estimator takes into account extra moment conditions from the level equation that rely on certain restrictions on the initial observations, which is arguably a better solution for endogeneity (Roodman, 2006). Therefore, this study applies the two-step system GMM estimator for empirical estimations. The GMM estimate requires a defined instrument (Ullah et al., 2020), which is quite challenging in empirical estimation. This study applies a standard procedure by using a lag of FD as an instrument for the FD, while all other factors are taking in one-year lags to eliminate any potential feedback effects from energy intensity to economic factors. Moreover, one by one the study adds control variables to check for the robustness of the results. As the sensitivity check, this study also employs other panel estimators including the feasible generalized least squares (FGLS) (Liao and Cao, 2013) and 
panel corrected standard errors model (PCSE) estimator (Bailey and Katz, 2011), which deal with heteroscedasticity and cross-sectional dependence, respectively.

In the second step, the study aims at investigating the influences of FD on energy intensity in the long run. The canonical correlation regression (CCR) and fully modified ordinary least squares (FMOLS) are recruited (Khodzhimatov, 2018). Third, the study takes a further step to investigate the role of FD in the energy use transformation in facing the changes in energy price. This study estimates the interaction term between each financial development indicator with the oil price and executes the predictive margins analysis for the impacts of oil prices on two levels of FD. Two levels of FD are measured by using the mean of each financial indicator to plus and minus one standard deviation, respectively, forming the low and high levels of FD. At last, the estimations for three subsamples are regressed by FGLS model and checked for the robustness by PCSE model since two-step system GMM estimator does not work well for the sample with two small sample size N ( subsample estimations, the study uses all independent variables in one-year lags to deal with endogeneity.

\section{Empirical results}

The results of our comprehensive empirical exercise are presented in Table 3 to Table 10. The robustness check by employing the FGLS model, FMOLS model and PCSE models are reported in Tables $A 3$ to $A 8$, Appendix, showing properly robust results. The results of twostep system GMM in Table 3 and Table 7 have insignificant test statistics of Hansen tests and Arellano-Bond test for autocorrelation (AR(2) test), which means two-step system GMM's results are consistent and unbiased (Roodman, 2006). Moreover, the robustness check by adding one by one control variables also suggests robustness of our results ${ }^{1}$.

\subsection{Financial development and Production energy intensity}

The estimates of FD's impact on the production energy intensity are presented in Table 3 to Table 6.

\footnotetext{
${ }^{1}$ The results of these estimations are provided upon requests.
} 
Table 3. Financial development and Production energy intensity

\begin{tabular}{|c|c|c|c|c|c|c|c|c|c|}
\hline Dep. var: PEI (GMM) & $(1)$ & $(2)$ & (3) & (4) & (5) & $(6)$ & $(7)$ & $(8)$ & (9) \\
\hline L.PEI & $\begin{array}{l}0.9524^{* * *} \\
{[0.0062]}\end{array}$ & $\begin{array}{l}0.9442^{* * *} \\
{[0.0025]}\end{array}$ & $\begin{array}{l}0.9300^{* * *} \\
{[0.0059]}\end{array}$ & $\begin{array}{l}0.9281^{* * *} \\
{[0.0032]}\end{array}$ & $\begin{array}{l}0.9312^{* * *} \\
{[0.0042]}\end{array}$ & $\begin{array}{l}0.9502^{* * *} \\
{[0.0052]}\end{array}$ & $\begin{array}{l}0.9277^{* * *} \\
{[0.0056]}\end{array}$ & $\begin{array}{l}0.9220^{* * *} \\
{[0.0071]}\end{array}$ & $\begin{array}{l}0.9358^{* * *} \\
{[0.0042]}\end{array}$ \\
\hline Income & $\begin{array}{l}-0.0169^{* * *} \\
{[0.0022]}\end{array}$ & $\begin{array}{l}-0.0171^{* * *} \\
{[0.0038]}\end{array}$ & $\begin{array}{l}-0.0165^{* * *} \\
{[0.0031]}\end{array}$ & $\begin{array}{l}-0.0139^{* * *} \\
{[0.0019]}\end{array}$ & $\begin{array}{l}-0.0089^{* * *} \\
{[0.0019]}\end{array}$ & $\begin{array}{l}-0.0148^{* * *} \\
{[0.0021]}\end{array}$ & $\begin{array}{l}-0.0174^{* * *} \\
{[0.0025]}\end{array}$ & $\begin{array}{l}-0.0145^{* * *} \\
{[0.0027]}\end{array}$ & $\begin{array}{l}-0.0146^{* *} \\
{[0.0019]}\end{array}$ \\
\hline Industry & $\begin{array}{l}-0.0004^{* * *} \\
{[0.0002]}\end{array}$ & $\begin{array}{l}-0.00001 \\
{[0.0002]}\end{array}$ & $\begin{array}{l}-0.0008^{* * *} \\
{[0.0003]}\end{array}$ & $\begin{array}{l}-0.0004 \\
{[0.0003]}\end{array}$ & $\begin{array}{l}-0.0001 \\
{[0.0002]}\end{array}$ & $\begin{array}{l}-0.0004 \\
{[0.0002]}\end{array}$ & $\begin{array}{l}-0.0005^{* *} \\
{[0.0002]}\end{array}$ & $\begin{array}{l}-0.0007^{* * *} \\
{[0.0002]}\end{array}$ & $\begin{array}{l}-0.0004 \\
{[0.0002]}\end{array}$ \\
\hline Trade & $\begin{array}{l}0.0002^{* * *} \\
{[0.00003]}\end{array}$ & $\begin{array}{l}0.0002^{* * *} \\
{[0.0000]}\end{array}$ & $\begin{array}{l}0.0002^{* * *} \\
{[0.00003]}\end{array}$ & $\begin{array}{l}0.0001^{*} \\
{[0.00005]}\end{array}$ & $\begin{array}{l}0.0001^{* * *} \\
{[0.00004]}\end{array}$ & $\begin{array}{l}0.0002^{* * *} \\
{[0.00003]}\end{array}$ & $\begin{array}{l}0.0001^{* * *} \\
{[0.00004]}\end{array}$ & $\begin{array}{l}0.0001^{* * *} \\
{[0.00004]}\end{array}$ & $\begin{array}{l}0.0002^{* * *} \\
{[0.00003]}\end{array}$ \\
\hline FDI & $\begin{array}{l}-0.0044^{* * *} \\
{[0.0006]}\end{array}$ & $\begin{array}{l}-0.0050^{* * *} \\
{[0.0005]}\end{array}$ & $\begin{array}{l}-0.0036^{* * *} \\
{[0.0005]}\end{array}$ & $\begin{array}{l}-0.0036^{* * *} \\
{[0.0005}\end{array}$ & $\begin{array}{l}-0.0041^{* * *} \\
{[0.0005]}\end{array}$ & $\begin{array}{l}-0.0041^{* * *} \\
{[0.0004]}\end{array}$ & $\begin{array}{l}-0.0030^{* * *} \\
{[0.0005]}\end{array}$ & $\begin{array}{l}-0.0029^{* * *} \\
{[0.0004]}\end{array}$ & $\begin{array}{l}-0.0043^{* * *} \\
{[0.0004]}\end{array}$ \\
\hline ES & $\begin{array}{l}0.0038^{* * *} \\
{[0.0009]}\end{array}$ & $\begin{array}{l}0.0039^{* * *} \\
{[0.0012]}\end{array}$ & $\begin{array}{l}0.0072^{* * *} \\
{[0.0012]}\end{array}$ & $\begin{array}{l}0.0068^{* * *} \\
{[0.0011]}\end{array}$ & $\begin{array}{l}0.0052^{* * *} \\
{[0.0010]}\end{array}$ & $\begin{array}{l}0.0041^{* * *} \\
{[0.0008]}\end{array}$ & $\begin{array}{l}0.0059^{* * *} \\
{[0.0011]}\end{array}$ & $\begin{array}{l}0.0067^{* * *} \\
{[0.0013]}\end{array}$ & $\begin{array}{l}0.0043^{* * *} \\
{[0.0010]}\end{array}$ \\
\hline $\mathrm{OP}$ & $\begin{array}{l}-0.0045^{* * *} \\
{[0.0016]}\end{array}$ & $\begin{array}{l}-0.0060^{* * *} \\
{[0.0018]}\end{array}$ & $\begin{array}{l}-0.0101^{* * *} \\
{[0.0014]}\end{array}$ & $\begin{array}{l}-0.0081^{* * *} \\
{[0.0012]}\end{array}$ & $\begin{array}{l}-0.0063^{* * *} \\
{[0.0015]}\end{array}$ & $\begin{array}{l}-0.0063^{* * *} \\
{[0.0012]}\end{array}$ & $\begin{array}{l}-0.0112^{* * *} \\
{[0.0014]}\end{array}$ & $\begin{array}{l}-0.0114^{* * *} \\
{[0.0015]}\end{array}$ & $\begin{array}{l}-0.0056^{* * *} \\
{[0.0014]}\end{array}$ \\
\hline OFD & $\begin{array}{l}\mathbf{0 . 0 6 0 3}{ }^{* * *} \\
{[0.0110]}\end{array}$ & & & & & & & & \\
\hline FI & & $\begin{array}{l}\mathbf{0 . 0 5 8 5} * \\
{[0.0270]}\end{array}$ & & & & & & & \\
\hline FID & & & $\begin{array}{l}\mathbf{0 . 0 2 2 7} \\
{[0.0135]}\end{array}$ & & & & & & \\
\hline FIA & & & & $\begin{array}{l}\mathbf{0 . 0 0 1 8} \\
{[0.0098]}\end{array}$ & & & & & \\
\hline FIE & & & & & $\begin{array}{l}-\mathbf{0 . 0 5 0 4} * * * \\
{[0.0146]}\end{array}$ & & & & \\
\hline FM & & & & & & $\begin{array}{l}\mathbf{0 . 0 4 3 4} * * * \\
{[0.0059]}\end{array}$ & & & \\
\hline FMD & & & & & & & $\begin{array}{l}\mathbf{0 . 0 3 7 4} * * * \\
{[0.0081]}\end{array}$ & & \\
\hline FMA & & & & & & & & $\begin{array}{l}\mathbf{0 . 0 0 3 5} \\
{[0.0069]}\end{array}$ & \\
\hline FME & & & & & & & & & $\begin{array}{l}\mathbf{0 . 0 2 3 9} * * * \\
{[0.0031]}\end{array}$ \\
\hline Constant & $\begin{array}{l}0.1946^{* * *} \\
{[0.0274]}\end{array}$ & $\begin{array}{l}0.2048^{* * *} \\
{[0.0242]}\end{array}$ & $\begin{array}{l}0.2558^{* * *} \\
{[0.0249]}\end{array}$ & $\begin{array}{l}0.2311^{* * *} \\
{[0.0190]}\end{array}$ & $\begin{array}{l}0.2141^{* * *} \\
{[0.0190]}\end{array}$ & $\begin{array}{l}0.1952^{* * *} \\
{[0.0245]}\end{array}$ & $\begin{array}{l}0.2677^{* * *} \\
{[0.0279]}\end{array}$ & $\begin{array}{l}0.2654^{* * *} \\
{[0.0328]}\end{array}$ & $\begin{array}{l}0.2211^{* * *} \\
{[0.0245]}\end{array}$ \\
\hline Observations & 1,296 & 1,296 & 1,296 & 1,296 & 1,296 & 1,296 & 1,296 & 1,296 & 1,296 \\
\hline No of country & 81 & 81 & 81 & 81 & 81 & 81 & 81 & 81 & 81 \\
\hline No of IVs & 66 & 66 & 66 & 66 & 66 & 66 & 66 & 66 & 66 \\
\hline $\mathrm{AR}(2)$ test (p-value) & 0.527 & 0.547 & 0.498 & 0.497 & 0.460 & 0.499 & 0.471 & 0.486 & 0.525 \\
\hline Hansen test ( $\mathrm{p}$-value) & 0.646 & 0.539 & 0.626 & 0.593 & 0.694 & 0.534 & 0.620 & 0.494 & 0.625 \\
\hline
\end{tabular}

Note: The two-step system GMM estimators; standard errors are in []; *, **,*** are significant levels at $10 \%, 5 \%$, and $1 \%$, respectively. 
Table 4. Financial development and Production energy intensity: long-term effects

\begin{tabular}{|c|c|c|c|c|c|c|c|c|c|}
\hline Dep. var: PEI (CCR) & (1) & $(2)$ & (3) & (4) & (5) & $(6)$ & (7) & $(8)$ & (9) \\
\hline Income & $\begin{array}{l}-0.3440^{* * *} \\
{[0.0622]}\end{array}$ & $\begin{array}{l}-0.4428^{* * *} \\
{[0.0609]}\end{array}$ & $\begin{array}{l}-0.4470^{* * *} \\
{[0.0748]}\end{array}$ & $\begin{array}{l}-0.5751^{* * *} \\
{[0.1099]}\end{array}$ & $\begin{array}{l}-0.2735^{* * *} \\
{[0.0564]}\end{array}$ & $\begin{array}{l}-0.2955^{* * *} \\
{[0.0654]}\end{array}$ & $\begin{array}{l}-0.2510^{* *} \\
{[0.1166]}\end{array}$ & $\begin{array}{l}-0.2500^{* * *} \\
{[0.0909]}\end{array}$ & $\begin{array}{l}-0.2276^{* * *} \\
{[0.0499]}\end{array}$ \\
\hline Industry & $\begin{array}{l}-0.0122^{* * *} \\
{[0.0014]}\end{array}$ & $\begin{array}{l}-0.0079^{* * *} \\
{[0.0013]}\end{array}$ & $\begin{array}{l}-0.0092^{* * *} \\
{[0.0015]}\end{array}$ & $\begin{array}{l}-0.0064^{* * *} \\
{[0.0019]}\end{array}$ & $\begin{array}{l}-0.0087^{* * *} \\
{[0.0011]}\end{array}$ & $\begin{array}{l}-0.0133^{* * *} \\
{[0.0015]}\end{array}$ & $\begin{array}{l}-0.0084^{* * *} \\
{[0.0016]}\end{array}$ & $\begin{array}{l}-0.0091^{* * *} \\
{[0.0017]}\end{array}$ & $\begin{array}{l}-0.0153^{* * *} \\
{[0.0014]}\end{array}$ \\
\hline Trade & $\begin{array}{l}-0.0001 \\
{[0.0005]}\end{array}$ & $\begin{array}{l}-0.0008 \\
{[0.0006]}\end{array}$ & $\begin{array}{l}-0.0004 \\
{[0.0007]}\end{array}$ & $\begin{array}{l}-0.0007 \\
{[0.0009]}\end{array}$ & $\begin{array}{l}-0.0010 \\
{[0.0007]}\end{array}$ & $\begin{array}{l}0.0001 \\
{[0.0005]}\end{array}$ & $\begin{array}{l}-0.0012 \\
{[0.0010]}\end{array}$ & $\begin{array}{l}-0.0010 \\
{[0.0007]}\end{array}$ & $\begin{array}{l}0.0002 \\
{[0.0004]}\end{array}$ \\
\hline FDI & $\begin{array}{l}-0.0103^{* * *} \\
{[0.0015]}\end{array}$ & $\begin{array}{l}-0.0003 \\
{[0.0008]}\end{array}$ & $\begin{array}{l}-0.0008 \\
{[0.0007]}\end{array}$ & $\begin{array}{l}0.0014 \\
{[0.0017]}\end{array}$ & $\begin{array}{l}0.0037^{* * *} \\
{[0.0009]}\end{array}$ & $\begin{array}{l}-0.0119^{* * *} \\
{[0.0018]}\end{array}$ & $\begin{array}{l}0.0027 \\
{[0.0020]}\end{array}$ & $\begin{array}{l}0.0015 \\
{[0.0034]}\end{array}$ & $\begin{array}{l}-0.0167^{* * *} \\
{[0.0016]}\end{array}$ \\
\hline $\mathrm{ES}$ & $\begin{array}{l}0.3572^{* * *} \\
{[0.0467]}\end{array}$ & $\begin{array}{l}0.3026^{* * *} \\
{[0.0404]}\end{array}$ & $\begin{array}{l}0.1808^{* * *} \\
{[0.0427]}\end{array}$ & $\begin{array}{l}0.3722^{* * *} \\
{[0.0424]}\end{array}$ & $\begin{array}{l}0.2970^{* * *} \\
{[0.0636]}\end{array}$ & $\begin{array}{l}0.3670^{* * *} \\
{[0.0502]}\end{array}$ & $\begin{array}{l}0.2967^{* * *} \\
{[0.0581]}\end{array}$ & $\begin{array}{l}0.2966^{* * *} \\
{[0.0622]}\end{array}$ & $\begin{array}{l}0.3372^{* * *} \\
{[0.0414]}\end{array}$ \\
\hline $\mathrm{OP}$ & $\begin{array}{l}-0.0615^{* * *} \\
{[0.0097]}\end{array}$ & $\begin{array}{l}-0.0118 \\
{[0.0095]}\end{array}$ & $\begin{array}{l}-0.0312^{* * *} \\
{[0.0100]}\end{array}$ & $\begin{array}{l}0.0152 \\
{[0.0129]}\end{array}$ & $\begin{array}{l}-0.0048 \\
{[0.0184]}\end{array}$ & $\begin{array}{l}-0.0713^{* * *} \\
{[0.0105]}\end{array}$ & $\begin{array}{l}-0.0097 \\
{[0.0142]}\end{array}$ & $\begin{array}{l}-0.0156 \\
{[0.0160]}\end{array}$ & $\begin{array}{l}-0.0699^{* * *} \\
{[0.0087]}\end{array}$ \\
\hline OFD & $\begin{array}{l}\mathbf{0 . 6 0 1 6} \\
{[0.0551]}\end{array}$ & & & & & & & & \\
\hline FI & & $\begin{array}{l}\text { 1.0051*** } \\
{[0.0953]}\end{array}$ & & & & & & & \\
\hline FID & & & $\begin{array}{l}\mathbf{1 . 1 4 7 9 * * *} \\
{[0.0632]}\end{array}$ & & & & & & \\
\hline FIA & & & & $\begin{array}{l}\mathbf{0 . 9 8 1 2}^{* * *} \\
{[0.2628]}\end{array}$ & & & & & \\
\hline FIE & & & & & $\begin{array}{l}\mathbf{- 0 . 1 0 3 4} \\
{[0.0956]}\end{array}$ & & & & \\
\hline FM & & & & & & $\begin{array}{l}\mathbf{0 . 3 6 8 8} * * * \\
{[0.0404]}\end{array}$ & & & \\
\hline FMD & & & & & & & $\begin{array}{l}\mathbf{0 . 0 3 3 4} \\
{[0.1011]}\end{array}$ & & \\
\hline FMA & & & & & & & & $\begin{array}{l}\mathbf{0 . 9 5 3 2} \\
{[2.0121]}\end{array}$ & \\
\hline FME & & & & & & & & & $\begin{array}{l}\mathbf{0 . 1 8 6 5} * * * \\
{[0.0136]}\end{array}$ \\
\hline Constant & $\begin{array}{l}2.5210^{* * *} \\
{[0.2597]}\end{array}$ & $\begin{array}{l}3.0683^{* * *} \\
{[0.3469]}\end{array}$ & $\begin{array}{l}4.0536^{* * *} \\
{[0.3257]}\end{array}$ & $\begin{array}{l}3.6327^{* * *} \\
{[0.5349]}\end{array}$ & $\begin{array}{l}2.3538^{* * *} \\
{[0.4101]}\end{array}$ & $\begin{array}{l}2.2785^{* * *} \\
{[0.2562]}\end{array}$ & $\begin{array}{l}2.1546^{* * *} \\
{[0.5182]}\end{array}$ & $\begin{array}{l}2.1762^{* * *} \\
{[0.3536]}\end{array}$ & $\begin{array}{l}2.0389^{* * *} \\
{[0.1892]}\end{array}$ \\
\hline Observations & 16 & 16 & 16 & 16 & 16 & 16 & 16 & 16 & 16 \\
\hline R-squared & 0.3705 & 0.4535 & 0.7474 & 0.3226 & 0.4891 & 0.3382 & 0.3536 & 0.3393 & 0.3897 \\
\hline
\end{tabular}

Note: Canonical correlation regression (CCR) estimators; standard errors are in []; *,**, *** are significant levels at $10 \%, 5 \%$, and 1\%,

respectively. 
First, Table 3 reports the influence of FD on production energy intensity showing that eight in nine indicators of FD have a significant positive impact on production energy intensity, while only financial institutions efficiency has a significant negative impact. These results imply that the higher FD would have increasing effects on energy intensity in production except for the higher efficiency in financial institutions. The results are checked by FGLS model showing consistent findings in Table A3, Appendix.

Next, the long-run effects of FD on production energy intensity are examined by the CCR model and presented in Table 4. The results in Table 4 show consistent findings that most of the financial indicators have positive impacts, while only financial institutions efficiency has a negative impact (even insignificant) in the long run. Interestingly, the long-run estimations show the statistical significance of overall financial development, financial institutions, financial institutions depth, financial institutions access, financial markets, and financial markets efficiency, which highlight the positive impacts of financial institutions in the longrun. The results are checked by FMOLS model and presented in Table A4, Appendix, showing consistent findings. 
Table 5. Financial development and Production energy intensity: the absorbing capability with the increases in energy price

\begin{tabular}{|c|c|c|c|c|c|c|c|c|c|}
\hline Dep. var: PEI (GMM) & $(1)$ & $(2)$ & $(3)$ & $(4)$ & (5) & $(6)$ & (7) & $(8)$ & (9) \\
\hline Indep. Var: FIN & OFD & $\mathrm{FI}$ & FID & FIA & FIE & FM & FMD & FMA & FME \\
\hline L.PEI & $\begin{array}{l}0.9244^{* * *} \\
{[0.0066]}\end{array}$ & $\begin{array}{l}0.9289^{* * *} \\
{[0.0047]}\end{array}$ & $\begin{array}{l}0.9129^{* * *} \\
{[0.0054]}\end{array}$ & $\begin{array}{l}0.9168^{* * *} \\
{[0.0058]}\end{array}$ & $\begin{array}{l}0.9255^{* * *} \\
{[0.0044]}\end{array}$ & $\begin{array}{l}0.9207^{* * *} \\
{[0.0060]}\end{array}$ & $\begin{array}{l}0.9127^{* * *} \\
{[0.0057]}\end{array}$ & $\begin{array}{l}0.9115^{* * *} \\
{[0.0082]}\end{array}$ & $\begin{array}{l}0.9238^{* * *} \\
{[0.0053]}\end{array}$ \\
\hline Income & $\begin{array}{l}-0.0240^{* * *} \\
{[0.0035]}\end{array}$ & $\begin{array}{l}-0.0269^{* * *} \\
{[0.0053]}\end{array}$ & $\begin{array}{l}-0.0202^{* * *} \\
{[0.0030]}\end{array}$ & $\begin{array}{l}-0.0191^{* * *} \\
{[0.0028]}\end{array}$ & $\begin{array}{l}-0.0125^{* * *} \\
{[0.0024]}\end{array}$ & $\begin{array}{l}-0.0191^{* * *} \\
{[0.0022]}\end{array}$ & $\begin{array}{l}-0.0220^{* * *} \\
{[0.0025]}\end{array}$ & $\begin{array}{l}-0.0194^{* * *} \\
{[0.0032]}\end{array}$ & $\begin{array}{l}-0.0153^{* * *} \\
{[0.0017]}\end{array}$ \\
\hline Industry & $\begin{array}{l}-0.0009^{* * *} \\
{[0.0003]}\end{array}$ & $\begin{array}{l}-0.0002 \\
{[0.0003]}\end{array}$ & $\begin{array}{l}-0.0005^{*} \\
{[0.0003]}\end{array}$ & $\begin{array}{l}-0.0006 \\
{[0.0004]}\end{array}$ & $\begin{array}{l}-0.0005 \\
{[0.0003]}\end{array}$ & $\begin{array}{l}-0.0011^{* * *} \\
{[0.0003]}\end{array}$ & $\begin{array}{l}-0.0008^{* *} \\
{[0.0003]}\end{array}$ & $\begin{array}{l}-0.0008^{* * *} \\
{[0.0003]}\end{array}$ & $\begin{array}{l}-0.0012^{* * *} \\
{[0.0003]}\end{array}$ \\
\hline Trade & $\begin{array}{l}0.0002^{* * *} \\
{[0.00008]}\end{array}$ & $\begin{array}{l}0.0001^{* * *} \\
{[0.00004]}\end{array}$ & $\begin{array}{l}0.0001^{* * *} \\
{[0.00004]}\end{array}$ & $\begin{array}{l}0.0002^{* * *} \\
{[0.00005]}\end{array}$ & $\begin{array}{l}0.0002^{* * *} \\
{[0.00004]}\end{array}$ & $\begin{array}{l}0.0003^{* * *} \\
{[0.00004]}\end{array}$ & $\begin{array}{l}0.0002^{* * *} \\
{[0.00005]}\end{array}$ & $\begin{array}{l}0.0002^{* * *} \\
{[0.00004]}\end{array}$ & $\begin{array}{l}0.0002^{* * *} \\
{[0.00004]}\end{array}$ \\
\hline FDI & $\begin{array}{l}-0.0036^{* * *} \\
{[0.0005]}\end{array}$ & $\begin{array}{l}-0.0036^{* * *} \\
{[0.0005]}\end{array}$ & $\begin{array}{l}-0.0034^{* * *} \\
{[0.0004]}\end{array}$ & $\begin{array}{l}-0.0028^{* * *} \\
{[0.0004]}\end{array}$ & $\begin{array}{l}-0.0039^{* * *} \\
{[0.0005]}\end{array}$ & $\begin{array}{l}-0.0039^{* * *} \\
{[0.0005]}\end{array}$ & $\begin{array}{l}-0.0032^{* * *} \\
{[0.0005]}\end{array}$ & $\begin{array}{l}-0.0040^{* * *} \\
{[0.0004]}\end{array}$ & $\begin{array}{l}-0.0035^{* * *} \\
{[0.0006]}\end{array}$ \\
\hline ES & $\begin{array}{l}0.0062^{* * *} \\
{[0.0013]}\end{array}$ & $\begin{array}{l}0.0051^{* * *} \\
{[0.0011]}\end{array}$ & $\begin{array}{l}0.0075^{* * *} \\
{[0.0012]}\end{array}$ & $\begin{array}{l}0.0072^{* * *} \\
{[0.0011]}\end{array}$ & $\begin{array}{l}0.0064^{* * *} \\
{[0.0012]}\end{array}$ & $\begin{array}{l}0.0076^{* * *} \\
{[0.0013]}\end{array}$ & $\begin{array}{l}0.0070^{* * *} \\
{[0.0012]}\end{array}$ & $\begin{array}{l}0.0074^{* * *} \\
{[0.0014]}\end{array}$ & $\begin{array}{l}0.0077^{* * *} \\
{[0.0010]}\end{array}$ \\
\hline $\mathbf{O P}$ & $\begin{array}{l}-0.0077^{* * *} \\
{[0.0029]}\end{array}$ & $\begin{array}{l}-\mathbf{0 . 0 0 4 8} \\
{[0.0029]}\end{array}$ & $\begin{array}{l}-0.0108 * * * \\
{[0.0028]}\end{array}$ & $\begin{array}{l}-0.0091 * * * \\
{[0.0024]}\end{array}$ & $\begin{array}{l}-0.0429 * * \\
{[0.0200]}\end{array}$ & $\begin{array}{l}-0.0089^{* * *} \\
{[0.0028]}\end{array}$ & $\begin{array}{l}-0.0099 * * * \\
{[0.0029]}\end{array}$ & $\begin{array}{l}-\mathbf{0 . 0 1 0 6 * * *} \\
{[0.0026]}\end{array}$ & $\begin{array}{l}-0.0110 * * * \\
{[0.0025]}\end{array}$ \\
\hline FIN & $\begin{array}{l}\mathbf{0 . 1 4 1 7} * * * \\
{[0.0232]}\end{array}$ & $\begin{array}{l}\mathbf{0 . 1 9 0 7} * * * \\
{[0.0413]}\end{array}$ & $\begin{array}{l}\mathbf{0 . 0 6 7 2} * * * \\
{[0.0254]}\end{array}$ & $\begin{array}{l}\mathbf{0 . 0 6 9 5 * * *} \\
{[0.0228]}\end{array}$ & $\begin{array}{l}-\mathbf{0 . 2 3 0 8} * * \\
{[0.1140]}\end{array}$ & $\begin{array}{l}\mathbf{0 . 0 6 7 8 * * *} \\
{[0.0203]}\end{array}$ & $\begin{array}{l}\text { 0.1241*** } \\
{[0.0167]}\end{array}$ & $\begin{array}{l}\mathbf{0 . 0 4 0 5 * *} \\
{[0.0179]}\end{array}$ & $\begin{array}{l}\mathbf{0 . 0 1 1 3} \\
{[0.0151]}\end{array}$ \\
\hline OP*FIN & $\begin{array}{l}-\mathbf{0 . 0 1 3 1} \\
{[0.0043]}\end{array}$ & $\begin{array}{l}-\mathbf{0 . 0 1 8 9 * * *} \\
{[0.0040]}\end{array}$ & $\begin{array}{l}-\mathbf{0 . 0 0 8 5} \\
{[0.0041]}\end{array}$ & $\begin{array}{l}-\mathbf{0 . 0 1 0 6} \\
{[0.0041]}\end{array}$ & $\begin{array}{l}\mathbf{0 . 0 5 5 1}^{*} \\
{[0.0304]}\end{array}$ & $\begin{array}{c}-\mathbf{0 . 0 0 8 0} \\
{[0.0048]}\end{array}$ & $\begin{array}{l}-\mathbf{0 . 0 1 8 2} \\
{[0.0041]}\end{array}$ & $\begin{array}{l}-\mathbf{0 . 0 0 2 8} \\
{[0.0043]}\end{array}$ & $\begin{array}{l}\mathbf{0 . 0 0 0 1} \\
{[0.0038]}\end{array}$ \\
\hline Constant & $\begin{array}{l}0.2945^{* * *} \\
{[0.0366]}\end{array}$ & $\begin{array}{l}0.2789^{* * *} \\
{[0.0371]}\end{array}$ & $\begin{array}{l}0.3077^{* * *} \\
{[0.0300]}\end{array}$ & $\begin{array}{l}0.2841^{* * *} \\
{[0.0251]}\end{array}$ & $\begin{array}{l}0.3719^{* * *} \\
{[0.0862]}\end{array}$ & $\begin{array}{l}0.2831^{* * *} \\
{[0.0305]}\end{array}$ & $\begin{array}{l}0.3184^{* * *} \\
{[0.0307]}\end{array}$ & $\begin{array}{l}0.3094^{* * *} \\
{[0.0411]}\end{array}$ & $\begin{array}{l}0.2652^{* * *} \\
{[0.0252]}\end{array}$ \\
\hline Observations & 1,296 & 1,296 & 1,296 & 1,296 & 1,296 & 1,296 & 1,296 & 1,296 & 1,296 \\
\hline No of country & 81 & 81 & 81 & 81 & 81 & 81 & 81 & 81 & 81 \\
\hline No of IVs & 68 & 68 & 68 & 68 & 65 & 68 & 68 & 68 & 68 \\
\hline $\mathrm{AR}(2)$ test (p-value) & 0.506 & 0.526 & 0.489 & 0.492 & 0.493 & 0.498 & 0.472 & 0.490 & 0.499 \\
\hline Hansen test (p-value) & 0.212 & 0.296 & 0.349 & 0.354 & 0.567 & 0.126 & 0.243 & 0.417 & 0.298 \\
\hline
\end{tabular}

Note: The two-step system GMM estimators; standard errors are in []; *, **, *** are significant levels at 10\%, 5\%, and 1\%, respectively. 


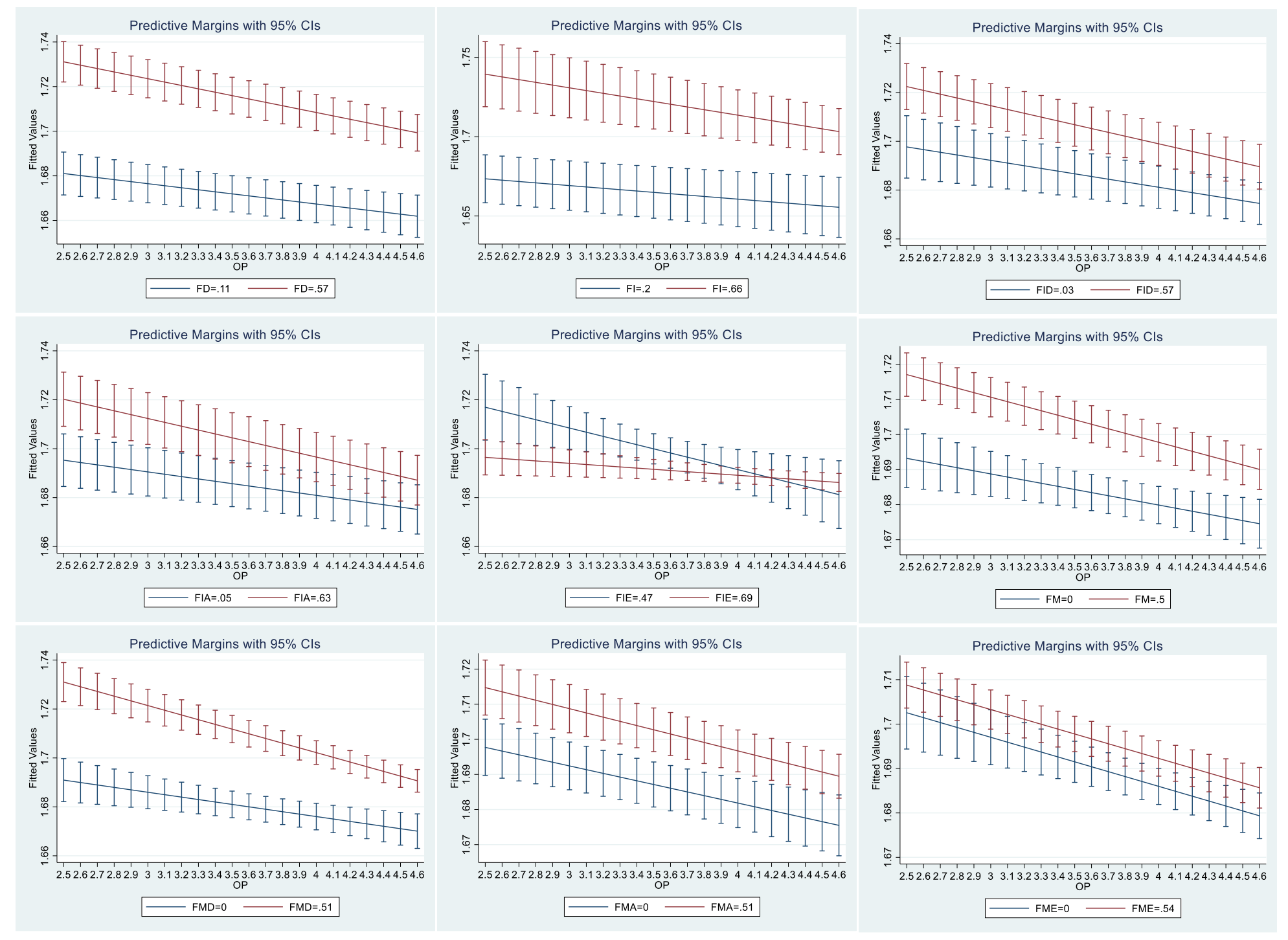

Figure 1. Predictive margins of the effects of oil price on Production Energy intensity by levels of financial development 
The estimation to investigate the role of FD in the face of higher energy prices through the interaction term between each financial indicator and oil price $(O P)$ is presented in Table 5. The results show that the oil price has a significant negative impact on production energy intensity, while the impacts of FD on production energy intensity are consistent with the results in Tables 3 and 4. That is, eight of nine financial indicators have significant positive impacts on production energy intensity, while only financial institutions efficiency has significant negative impacts. Interestingly, the interaction terms between each financial indicator with oil price mostly have negative impacts. This implies that the higher oil price helps to reduce energy intensity in production, which is stronger in the case of countries with higher FD. Likewise, FD is likely a good catalyst for energy transformation toward higher efficiency in facing higher energy costs. The predictive margins analysis for the impacts of oil price on production energy intensity in two levels of FD are illustrated in Figure 1 shows a clearer picture. That is, the slopes of the impacts of oil prices are likely to be larger (the line is steeper) in the countries with the high level of FD, which confirm the good catalyst role of FD in transforming the energy consumption for production toward the higher efficiency under the pressure of higher energy price.

At last, the influences of FD on production energy intensity are examined for three subsamples and presented in Table 6. In LMEs, there are mixed impacts of FD on production energy intensity. Financial institutions access has significant positive impacts, while financial institutions efficiency, financial markets, financial market depth, and financial market efficiency have significant negative impacts. Others have insignificant impacts. The results mean that the FD generally reduces the energy intensity for production in LMEs. In UMEs, most of the financial indicators have significant positive impacts except the negative impact of financial markets access. This means that the FD generally induces higher production energy intensity in UMEs. In HIEs, most of the financial indicators have significant negative impacts on production energy intensity except the financial institution's efficiency with positive impact but statistical insignificance. Overall, the results show a heteroscedastic effect of FD on production energy intensity across income levels. The FD helps to reduce production energy intensity in LMEs and HIEs, while it induces higher production energy intensity in UMEs. 
Table 6. Financial development and Production energy intensity: three subsamples

\begin{tabular}{|c|c|c|c|c|c|c|c|c|c|}
\hline LMES & & & & & & & & & \\
\hline Dep. var: PEI (FGLS) & $(1)$ & $(2)$ & (3) & $(4)$ & (5) & $(6)$ & (7) & $(8)$ & (9) \\
\hline Indep. Var: FIN & OFD & FI & FID & FIA & FIE & FM & FMD & FMA & FME \\
\hline Income $(-1)$ & $\begin{array}{l}-0.4798^{* * *} \\
{[0.0393]}\end{array}$ & $\begin{array}{l}-0.5089^{* * *} \\
{[0.0444]}\end{array}$ & $\begin{array}{l}-0.4924^{* * *} \\
{[0.0426]}\end{array}$ & $\begin{array}{l}-0.6576^{* * *} \\
{[0.0396]}\end{array}$ & $\begin{array}{l}-0.4536^{* * *} \\
{[0.0366]}\end{array}$ & $\begin{array}{l}-0.4918^{* * *} \\
{[0.0373]}\end{array}$ & $\begin{array}{l}-0.4880^{* * *} \\
{[0.0375]}\end{array}$ & $\begin{array}{l}-0.4863^{* * *} \\
{[0.0408]}\end{array}$ & $\begin{array}{l}-0.5070^{* * *} \\
{[0.0373]}\end{array}$ \\
\hline Industry $(-1)$ & $\begin{array}{l}-0.0180^{* * *} \\
{[0.0035]}\end{array}$ & $\begin{array}{l}-0.0185^{* * *} \\
{[0.0035]}\end{array}$ & $\begin{array}{l}-0.0189^{* * *} \\
{[0.0035]}\end{array}$ & $\begin{array}{c}-0.0067^{*} \\
{[0.0036]}\end{array}$ & $\begin{array}{l}-0.0146^{* * *} \\
{[0.0034]}\end{array}$ & $\begin{array}{l}-0.0165^{* * *} \\
{[0.0036]}\end{array}$ & $\begin{array}{l}-0.0166^{* * *} \\
{[0.0035]}\end{array}$ & $\begin{array}{l}-0.0172^{* * *} \\
{[0.0039]}\end{array}$ & $\begin{array}{l}-0.0180^{* * *} \\
{[0.0035]}\end{array}$ \\
\hline Trade $(-1)$ & $\begin{array}{l}0.0049^{* * *} \\
{[0.0008]}\end{array}$ & $\begin{array}{l}0.0049^{* * *} \\
{[0.0008]}\end{array}$ & $\begin{array}{l}0.0049 * * * \\
{[0.0008]}\end{array}$ & $\begin{array}{l}0.0033^{* * *} \\
{[0.0008]}\end{array}$ & $\begin{array}{l}0.0049^{* * *} \\
{[0.0008]}\end{array}$ & $\begin{array}{l}0.0047^{* * *} \\
{[0.0008]}\end{array}$ & $\begin{array}{l}0.0044^{* * *} \\
{[0.0009]}\end{array}$ & $\begin{array}{l}0.0047^{* * *} \\
{[0.0009]}\end{array}$ & $\begin{array}{l}0.0051^{* * *} \\
{[0.0008]}\end{array}$ \\
\hline FDI $(-1)$ & $\begin{array}{l}-0.0048 \\
{[0.0053]}\end{array}$ & $\begin{array}{l}-0.0032 \\
{[0.0054]}\end{array}$ & $\begin{array}{l}-0.0039 \\
{[0.0054]}\end{array}$ & $\begin{array}{l}0.0005 \\
{[0.0050]}\end{array}$ & $\begin{array}{l}-0.0072 \\
{[0.0051]}\end{array}$ & $\begin{array}{l}-0.0046 \\
{[0.0053]}\end{array}$ & $\begin{array}{l}-0.0039 \\
{[0.0052]}\end{array}$ & $\begin{array}{l}-0.0037 \\
{[0.0053]}\end{array}$ & $\begin{array}{l}-0.0045 \\
{[0.0053]}\end{array}$ \\
\hline $\mathrm{ES}(-1)$ & $\begin{array}{l}0.1725^{* * *} \\
{[0.0123]}\end{array}$ & $\begin{array}{l}0.1657^{* * *} \\
{[0.0123]}\end{array}$ & $\begin{array}{l}0.1672^{* * *} \\
{[0.0120]}\end{array}$ & $\begin{array}{l}0.1255^{* * *} \\
{[0.0122]}\end{array}$ & $\begin{array}{l}0.1639^{* * *} \\
{[0.0114]}\end{array}$ & $\begin{array}{l}0.1734^{* * *} \\
{[0.0122]}\end{array}$ & $\begin{array}{l}0.1726^{* * *} \\
{[0.0120]}\end{array}$ & $\begin{array}{l}0.1646^{* * *} \\
{[0.0121]}\end{array}$ & $\begin{array}{l}0.1749^{* * *} \\
{[0.0125]}\end{array}$ \\
\hline $\mathrm{OP}$ & $\begin{array}{l}-0.1348^{* * *} \\
{[0.0367]}\end{array}$ & $\begin{array}{l}-0.1426^{* * *} \\
{[0.0375]}\end{array}$ & $\begin{array}{l}-0.1367^{* * *} \\
{[0.0375]}\end{array}$ & $\begin{array}{l}-0.1824^{* * *} \\
{[0.0347]}\end{array}$ & $\begin{array}{l}-0.1213^{* * *} \\
{[0.0353]}\end{array}$ & $\begin{array}{l}-0.1403^{* * *} \\
{[0.0364]}\end{array}$ & $\begin{array}{l}-0.1243^{* * *} \\
{[0.0369]}\end{array}$ & $\begin{array}{l}-0.1406^{* * *} \\
{[0.0366]}\end{array}$ & $\begin{array}{l}-0.1449^{* * *} \\
{[0.0366]}\end{array}$ \\
\hline FIN $(-1)$ & $\begin{array}{l}-\mathbf{0 . 4 3 9 9 *} \\
{[0.2578]}\end{array}$ & $\begin{array}{l}\mathbf{0 . 1 0 0 4} \\
{[0.3253]}\end{array}$ & $\begin{array}{l}-\mathbf{0 . 1 4 9 3} \\
{[0.3382]}\end{array}$ & $\begin{array}{l}1.7323^{* * *} \\
{[0.2070]}\end{array}$ & $\begin{array}{l}-0.9447^{* * *} \\
{[0.1441]}\end{array}$ & $\begin{array}{l}-\mathbf{0 . 4 1 1 5 * *} \\
{[0.1707]}\end{array}$ & $\begin{array}{l}-\mathbf{0 . 5 9 6 1 * *} \\
{[0.2324]}\end{array}$ & $\begin{array}{l}\mathbf{- 0 . 1 8 9 9} \\
{[0.2069]}\end{array}$ & $\begin{array}{l}-\mathbf{0 . 1 5 4 0 *} \\
{[0.0741]}\end{array}$ \\
\hline Constant & $\begin{array}{l}5.2273^{* * *} \\
{[0.2427]}\end{array}$ & $\begin{array}{l}5.3917^{* * *} \\
{[0.2603]}\end{array}$ & $\begin{array}{l}5.2967^{* * *} \\
{[0.2667]}\end{array}$ & $\begin{array}{l}6.3731^{* * *} \\
{[0.2486]}\end{array}$ & $\begin{array}{l}5.4042^{* * *} \\
{[0.2223]}\end{array}$ & $\begin{array}{l}5.2689^{* * *} \\
{[0.2330]}\end{array}$ & $\begin{array}{l}5.2072^{* * *} \\
{[0.2372]}\end{array}$ & $\begin{array}{l}5.2545^{* * *} \\
{[0.2560]}\end{array}$ & $\begin{array}{l}5.3832^{* * *} \\
{[0.2310]}\end{array}$ \\
\hline Observations & 496 & 496 & 496 & 496 & 496 & 496 & 496 & 496 & 496 \\
\hline No of Country & 31 & 31 & 31 & 31 & 31 & 31 & 31 & 31 & 31 \\
\hline \multicolumn{10}{|l|}{ UMEs } \\
\hline Dep. var: PEI (FGLS) & $(1)$ & $(2)$ & (3) & $(4)$ & $(5)$ & $(6)$ & (7) & $(8)$ & (9) \\
\hline Indep. Var: FIN & OFD & FI & FID & FIA & FIE & FM & FMD & FMA & FME \\
\hline Income $(-1)$ & $\begin{array}{l}-0.3348^{* * *} \\
{[0.0532]}\end{array}$ & $\begin{array}{l}-0.3063^{* * *} \\
{[0.0526]}\end{array}$ & $\begin{array}{l}-0.2908^{* * *} \\
{[0.0521]}\end{array}$ & $\begin{array}{l}-0.2877^{* * *} \\
{[0.0562]}\end{array}$ & $\begin{array}{l}-0.2454^{* * *} \\
{[0.0520]}\end{array}$ & $\begin{array}{l}-0.3243^{* * *} \\
{[0.0531]}\end{array}$ & $\begin{array}{l}-0.3478^{* * *} \\
{[0.0515]}\end{array}$ & $\begin{array}{l}-0.2301^{* * *} \\
{[0.0547]}\end{array}$ & $\begin{array}{l}-0.3055^{* * *} \\
{[0.0509]}\end{array}$ \\
\hline Industry $(-1)$ & $\begin{array}{l}-0.0027 \\
{[0.0028]}\end{array}$ & $\begin{array}{l}0.0003 \\
{[0.0029]}\end{array}$ & $\begin{array}{l}-0.0011 \\
{[0.0029]}\end{array}$ & $\begin{array}{l}0.0005 \\
{[0.0033]}\end{array}$ & $\begin{array}{l}-0.0028 \\
{[0.0029]}\end{array}$ & $\begin{array}{l}-0.0041 \\
{[0.0029]}\end{array}$ & $\begin{array}{l}-0.0041 \\
{[0.0028]}\end{array}$ & $\begin{array}{l}0.0001 \\
{[0.0029]}\end{array}$ & $\begin{array}{l}-0.0052^{*} \\
{[0.0029]}\end{array}$ \\
\hline Trade $(-1)$ & $\begin{array}{l}0.0022^{* * *} \\
{[0.0005]}\end{array}$ & $\begin{array}{l}0.0019^{* * *} \\
{[0.0006]}\end{array}$ & $\begin{array}{l}0.0021^{* * *} \\
{[0.0006]}\end{array}$ & $\begin{array}{l}0.0025^{* * *} \\
{[0.0006]}\end{array}$ & $\begin{array}{l}0.0022^{* * *} \\
{[0.0006]}\end{array}$ & $\begin{array}{l}0.0026^{* * *} \\
{[0.0005]}\end{array}$ & $\begin{array}{l}0.0016^{* * *} \\
{[0.0005]}\end{array}$ & $\begin{array}{l}0.0025^{* * *} \\
{[0.0005]}\end{array}$ & $\begin{array}{l}0.0032^{* * *} \\
{[0.0005]}\end{array}$ \\
\hline FDI $(-1)$ & $\begin{array}{l}-0.0021 \\
{[0.0057]}\end{array}$ & $\begin{array}{l}-0.0031 \\
{[0.0058]}\end{array}$ & $\begin{array}{l}-0.0001 \\
{[0.0057]}\end{array}$ & $\begin{array}{l}-0.0015 \\
{[0.0060]}\end{array}$ & $\begin{array}{l}-0.0027 \\
{[0.0058]}\end{array}$ & $\begin{array}{l}-0.0004 \\
{[0.0057]}\end{array}$ & $\begin{array}{l}0.0014 \\
{[0.0055]}\end{array}$ & $\begin{array}{l}-0.0003 \\
{[0.0058]}\end{array}$ & $\begin{array}{l}-0.0024 \\
{[0.0056]}\end{array}$ \\
\hline $\mathrm{ES}(-1)$ & $\begin{array}{l}0.0720^{* * *} \\
{[0.0160]}\end{array}$ & $\begin{array}{l}0.0709^{* * *} \\
{[0.0164]}\end{array}$ & $\begin{array}{l}0.0788^{* * *} \\
{[0.0160]}\end{array}$ & $\begin{array}{l}0.0799^{* * *} \\
{[0.0168]}\end{array}$ & $\begin{array}{l}0.0798^{* * *} \\
{[0.0160]}\end{array}$ & $\begin{array}{l}0.0789^{* * *} \\
{[0.0158]}\end{array}$ & $\begin{array}{l}0.0741^{* * *} \\
{[0.0154]}\end{array}$ & $\begin{array}{l}0.0863^{* * *} \\
{[0.0160]}\end{array}$ & $\begin{array}{l}0.0784^{* * *} \\
{[0.0156]}\end{array}$ \\
\hline $\mathrm{OP}$ & $\begin{array}{l}-0.1098^{* * *} \\
{[0.0375]}\end{array}$ & $\begin{array}{l}-0.1247^{* * *} \\
{[0.0386]}\end{array}$ & $\begin{array}{l}-0.1044^{* * *} \\
{[0.0380]}\end{array}$ & $\begin{array}{l}-0.1075^{* * *} \\
{[0.0392]}\end{array}$ & $\begin{array}{l}-0.1239^{* * *} \\
{[0.0390]}\end{array}$ & $\begin{array}{l}-0.0940^{* *} \\
{[0.0376]}\end{array}$ & $\begin{array}{l}-0.1147^{* * *} \\
{[0.0367]}\end{array}$ & $\begin{array}{l}-0.1033^{* * *} \\
{[0.0382]}\end{array}$ & $\begin{array}{l}-0.0811^{* *} \\
{[0.0373]}\end{array}$ \\
\hline FIN $(-1)$ & $\begin{array}{l}\mathbf{0 . 6 0 3 1} * * * \\
{[0.1436]}\end{array}$ & $\begin{array}{l}\mathbf{0 . 5 2 6 5} \text { *** } \\
{[0.1613]}\end{array}$ & $\begin{array}{l}\mathbf{0 . 2 5 7 2} * * * \\
{[0.0930]}\end{array}$ & $\begin{array}{l}\mathbf{0 . 1 4 9 7} \\
{[0.1499]}\end{array}$ & $\begin{array}{l}\mathbf{0 . 3 7 0 8 * * *} \\
{[0.1358]}\end{array}$ & $\begin{array}{l}\mathbf{0 . 3 9 0 0} \text { *** } \\
{[0.1028]}\end{array}$ & $\begin{array}{l}\mathbf{0 . 5 7 7 0 * * *} \\
{[0.0996]}\end{array}$ & $\begin{array}{l}-\mathbf{0 . 2 0 4 9 * *} \\
{[0.1024]}\end{array}$ & $\begin{array}{l}\mathbf{0 . 3 1 0 1 * * *} \\
{[0.0636]}\end{array}$ \\
\hline Constant & $\begin{array}{l}4.1658^{* * *} \\
{[0.4183]}\end{array}$ & $\begin{array}{l}3.9059^{* * *} \\
{[0.4106]}\end{array}$ & $\begin{array}{l}3.8012^{* * *} \\
{[0.4078]}\end{array}$ & $\begin{array}{l}3.7318^{* * *} \\
{[0.4358]}\end{array}$ & $\begin{array}{l}3.3910^{* * *} \\
{[0.4041]}\end{array}$ & $\begin{array}{l}4.0815^{* * *} \\
{[0.4178]}\end{array}$ & $\begin{array}{l}4.4327^{* * *} \\
{[0.4128]}\end{array}$ & $\begin{array}{l}3.2739^{* * *} \\
{[0.4267]}\end{array}$ & $\begin{array}{l}3.8674^{* * *} \\
{[0.3947]}\end{array}$ \\
\hline Observations & 336 & 336 & 336 & 336 & 336 & 336 & 336 & 336 & 336 \\
\hline
\end{tabular}




\begin{tabular}{|c|c|c|c|c|c|c|c|c|c|}
\hline No of Country & 21 & 21 & 21 & 21 & 21 & 21 & 21 & 21 & 21 \\
\hline \multicolumn{10}{|l|}{ HIEs } \\
\hline Dep. var: PEI (FGLS) & (1) & (2) & (3) & $(4)$ & $(5)$ & (6) & (7) & (8) & (9) \\
\hline Indep. Var: FIN & OFD & FI & FID & FIA & FIE & FM & FMD & FMA & FME \\
\hline Income(-1) & $\begin{array}{l}0.0455 \\
{[0.0389]}\end{array}$ & $\begin{array}{l}0.1237^{* * *} \\
{[0.0372]}\end{array}$ & $\begin{array}{l}0.0824^{* *} \\
{[0.0392]}\end{array}$ & $\begin{array}{l}0.0172 \\
{[0.0247]}\end{array}$ & $\begin{array}{l}-0.0729^{* * *} \\
{[0.0236]}\end{array}$ & $\begin{array}{l}-0.0464 \\
{[0.0316]}\end{array}$ & $\begin{array}{l}-0.0556^{*} \\
{[0.0305]}\end{array}$ & $\begin{array}{l}0.0070 \\
{[0.0227]}\end{array}$ & $\begin{array}{l}-0.1167^{* * *} \\
{[0.0235]}\end{array}$ \\
\hline Industry $(-1)$ & $\begin{array}{l}0.0160^{* * *} \\
{[0.0028]}\end{array}$ & $\begin{array}{l}0.0137^{* * *} \\
{[0.0028]}\end{array}$ & $\begin{array}{l}0.0125^{* * *} \\
{[0.0029]}\end{array}$ & $\begin{array}{l}0.0150^{* * *} \\
{[0.0028]}\end{array}$ & $\begin{array}{l}0.0156^{* * *} \\
{[0.0029]}\end{array}$ & $\begin{array}{l}0.0162^{* * *} \\
{[0.0029]}\end{array}$ & $\begin{array}{l}0.0160^{* * *} \\
{[0.0029]}\end{array}$ & $\begin{array}{l}0.0185^{* * *} \\
{[0.0028]}\end{array}$ & $\begin{array}{l}0.0147^{* * *} \\
{[0.0028]}\end{array}$ \\
\hline Trade $(-1)$ & $\begin{array}{l}-0.0008^{*} \\
{[0.0004]}\end{array}$ & $\begin{array}{l}-0.0006 \\
{[0.0004]}\end{array}$ & $\begin{array}{l}-0.0006 \\
{[0.0004]}\end{array}$ & $\begin{array}{l}-0.0007^{*} \\
{[0.0004]}\end{array}$ & $\begin{array}{l}-0.0006 \\
{[0.0004]}\end{array}$ & $\begin{array}{l}-0.0006 \\
{[0.0004]}\end{array}$ & $\begin{array}{l}-0.0005 \\
{[0.0004]}\end{array}$ & $\begin{array}{l}-0.0005 \\
{[0.0004]}\end{array}$ & $\begin{array}{l}-0.0001 \\
{[0.0004]}\end{array}$ \\
\hline FDI $(-1)$ & $\begin{array}{l}-0.0020 \\
{[0.0026]}\end{array}$ & $\begin{array}{l}-0.0028 \\
{[0.0025]}\end{array}$ & $\begin{array}{l}-0.0008 \\
{[0.0026]}\end{array}$ & $\begin{array}{l}-0.0043^{*} \\
{[0.0026]}\end{array}$ & $\begin{array}{l}-0.0030 \\
{[0.0026]}\end{array}$ & $\begin{array}{l}-0.0029 \\
{[0.0027]}\end{array}$ & $\begin{array}{l}-0.0030 \\
{[0.0027]}\end{array}$ & $\begin{array}{l}-0.0022 \\
{[0.0025]}\end{array}$ & $\begin{array}{l}-0.0041 \\
{[0.0026]}\end{array}$ \\
\hline $\mathrm{ES}(-1)$ & $\begin{array}{l}0.0231^{* *} \\
{[0.0098]}\end{array}$ & $\begin{array}{l}0.0139 \\
{[0.0096]}\end{array}$ & $\begin{array}{l}0.0216^{* *} \\
{[0.0097]}\end{array}$ & $\begin{array}{l}0.0154 \\
{[0.0097]}\end{array}$ & $\begin{array}{l}0.0226^{* *} \\
{[0.0100]}\end{array}$ & $\begin{array}{l}0.0223^{* *} \\
{[0.0100]}\end{array}$ & $\begin{array}{l}0.0218^{* *} \\
{[0.0100]}\end{array}$ & $\begin{array}{l}0.0296^{* * *} \\
{[0.0096]}\end{array}$ & $\begin{array}{l}0.0207^{* *} \\
{[0.0097]}\end{array}$ \\
\hline $\mathrm{OP}$ & $\begin{array}{l}-0.1066^{* * *} \\
{[0.0241]}\end{array}$ & $\begin{array}{l}-0.0941^{* * *} \\
{[0.0237]}\end{array}$ & $\begin{array}{l}-0.1170^{* * *} \\
{[0.0239]}\end{array}$ & $\begin{array}{l}-0.0928^{* * *} \\
{[0.0240]}\end{array}$ & $\begin{array}{l}-0.1144^{* * *} \\
{[0.0245]}\end{array}$ & $\begin{array}{l}-0.1117^{* * *} \\
{[0.0244]}\end{array}$ & $\begin{array}{l}-0.1110^{* * *} \\
{[0.0245]}\end{array}$ & $\begin{array}{l}-0.1126^{* * *} \\
{[0.0235]}\end{array}$ & $\begin{array}{l}-0.1045^{* * *} \\
{[0.0240]}\end{array}$ \\
\hline FIN(-1) & $\begin{array}{l}-\mathbf{0 . 4 0 5 3} 3^{* * *} \\
{[0.1261]}\end{array}$ & $\begin{array}{l}-0.7600^{* * *} \\
{[0.1300]}\end{array}$ & $\begin{array}{l}-0.4330^{* * *} \\
{[0.1014]}\end{array}$ & $\begin{array}{l}-\mathbf{0 . 3 5 5 1 * * *} \\
{[0.0683]}\end{array}$ & $\begin{array}{l}\mathbf{0 . 1 1 0 2} \\
{[0.1290]}\end{array}$ & $\begin{array}{l}-\mathbf{0 . 0 5 6 3} \\
{[0.0866]}\end{array}$ & $\begin{array}{l}\mathbf{- 0 . 0 2 0 8} \\
{[0.0715]}\end{array}$ & $\begin{array}{l}-\mathbf{0 . 3 0 5 1} \\
{[0.0524]}\end{array}$ & $\begin{array}{l}\mathbf{0 . 1 8 9 4}^{* * * *} \\
{[0.0448]}\end{array}$ \\
\hline Constant & $\begin{array}{l}1.2521^{* * *} \\
{[0.3451]}\end{array}$ & $\begin{array}{l}0.7961^{* *} \\
{[0.3093]}\end{array}$ & $\begin{array}{l}0.9972^{* * *} \\
{[0.3387]}\end{array}$ & $\begin{array}{l}1.5541^{* * *} \\
{[0.2386]}\end{array}$ & $\begin{array}{l}2.1624^{* * *} \\
{[0.2253]}\end{array}$ & $\begin{array}{l}1.9766^{* * *} \\
{[0.3067]}\end{array}$ & $\begin{array}{l}2.0569^{* * *} \\
{[0.2992]}\end{array}$ & $\begin{array}{l}1.4455^{* * *} \\
{[0.2407]}\end{array}$ & $\begin{array}{l}2.5502^{* * *} \\
{[0.2380]}\end{array}$ \\
\hline Observations & 464 & 464 & 464 & 464 & 464 & 464 & 464 & 464 & 464 \\
\hline No of Country & 29 & 29 & 29 & 29 & 29 & 29 & 29 & 29 & 29 \\
\hline
\end{tabular}

Note: Feasible generalized least square (FGLS) estimators; standard errors are in []; ***,***are significant levels at 10\%, 5\%, and $1 \%$, respectively. 


\subsection{Financial development and Consumption energy intensity}

The results on the influence of FD on consumption energy intensity are presented in Tables 7 to Tables 10. The robustness checks by employing other estimation approaches are reported in Table A6 to A8, Appendix. Table 7 presents the influences of FD on consumption energy intensity showing that financial institutions, financial institutions depth, financial institutions access, financial markets depth, and financial markets access have a significant negative impact, while financial institutions efficiency and financial market efficiency have a significant positive impact. The results mean that the financial depth and financial access (in both financial institutions and financial markets) reduce the energy intensity in citizens' consumption, while the financial efficiency increases it.

Table 8 reports the long-term effects of FD on consumption energy intensity. It is interesting to notice that the long-run effects of FD on consumption energy intensity are quietly different from the results in Table 7. The results in Table 8 show that financial institutions and its three dimensions (depth, access, and efficiency) have a significant positive impact on consumption energy intensity, while financial markets and its three dimensions (depth, access, and efficiency) have a negative but insignificant impact. This means that the financial institutions and its dimensions are important for consumption energy intensity in the long run as the inducing factor. 
Table 7. Financial development and Consumption energy intensity

\begin{tabular}{|c|c|c|c|c|c|c|c|c|c|}
\hline Dep. var: CEI (GMM) & $(1)$ & $(2)$ & (3) & $(4)$ & (5) & $(6)$ & $(7)$ & $(8)$ & $(9)$ \\
\hline L.CEI & $\begin{array}{l}0.9659^{* * *} \\
{[0.0035]}\end{array}$ & $\begin{array}{l}0.9609^{* * *} \\
{[0.0031]}\end{array}$ & $\begin{array}{l}0.9656^{* * *} \\
{[0.0037]}\end{array}$ & $\begin{array}{l}0.9679^{* * *} \\
{[0.0027]}\end{array}$ & $\begin{array}{l}0.9559^{* * *} \\
{[0.0019]}\end{array}$ & $\begin{array}{l}0.9581^{* * *} \\
{[0.0027]}\end{array}$ & $\begin{array}{l}0.9709^{* * *} \\
{[0.0028]}\end{array}$ & $\begin{array}{l}0.9674^{* * *} \\
{[0.0028]}\end{array}$ & $\begin{array}{l}0.9509^{* * *} \\
{[0.0030]}\end{array}$ \\
\hline Income & $\begin{array}{l}0.0078^{* * *} \\
{[0.0029]}\end{array}$ & $\begin{array}{l}0.0182^{* * *} \\
{[0.0031]}\end{array}$ & $\begin{array}{l}0.0148^{* * *} \\
{[0.0023]}\end{array}$ & $\begin{array}{l}0.0169^{* * *} \\
{[0.0022]}\end{array}$ & $\begin{array}{l}0.0018 \\
{[0.0017]}\end{array}$ & $\begin{array}{l}0.0013 \\
{[0.0021]}\end{array}$ & $\begin{array}{l}0.0130^{* * *} \\
{[0.0018]}\end{array}$ & $\begin{array}{l}0.0065^{* * *} \\
{[0.0015]}\end{array}$ & $\begin{array}{l}0.0133^{* * *} \\
{[0.0021]}\end{array}$ \\
\hline Urban & $\begin{array}{l}0.0003^{* * *} \\
{[0.0001]}\end{array}$ & $\begin{array}{l}0.0003^{* *} \\
{[0.0001]}\end{array}$ & $\begin{array}{l}0.0003^{* * *} \\
{[0.0001]}\end{array}$ & $\begin{array}{l}0.0002 \\
{[0.0001]}\end{array}$ & $\begin{array}{l}0.0008^{* * *} \\
{[0.0001]}\end{array}$ & $\begin{array}{l}0.0006^{* * *} \\
{[0.0001]}\end{array}$ & $\begin{array}{l}0.0002^{* *} \\
{[0.0001]}\end{array}$ & $\begin{array}{l}0.0002^{* *} \\
{[0.0001]}\end{array}$ & $\begin{array}{l}0.0007^{* * *} \\
{[0.0001]}\end{array}$ \\
\hline Trade & $\begin{array}{l}0.0003^{* * *} \\
{[0.00003]}\end{array}$ & $\begin{array}{l}0.0003^{* * *} \\
{[0.00005]}\end{array}$ & $\begin{array}{l}0.0003^{* * *} \\
{[0.00004]}\end{array}$ & $\begin{array}{l}0.0002^{* * *} \\
{[0.00003]}\end{array}$ & $\begin{array}{l}0.0002^{* * *} \\
{[0.00003]}\end{array}$ & $\begin{array}{l}0.0004^{* * *} \\
{[0.00003]}\end{array}$ & $\begin{array}{l}0.0001^{* * *} \\
{[0.00002]}\end{array}$ & $\begin{array}{l}0.0002^{* * *} \\
{[0.00002]}\end{array}$ & $\begin{array}{l}0.0004^{* * *} \\
{[0.00003]}\end{array}$ \\
\hline ES & $\begin{array}{l}0.0103^{* * *} \\
{[0.0008]}\end{array}$ & $\begin{array}{l}0.0103^{* * *} \\
{[0.0008]}\end{array}$ & $\begin{array}{l}0.0089^{* * *} \\
{[0.0007]}\end{array}$ & $\begin{array}{l}0.0110^{* * *} \\
{[0.0008]}\end{array}$ & $\begin{array}{l}0.0126^{* * *} \\
{[0.0005]}\end{array}$ & $\begin{array}{l}0.0124^{* * *} \\
{[0.0009]}\end{array}$ & $\begin{array}{l}0.0089^{* * *} \\
{[0.0008]}\end{array}$ & $\begin{array}{l}0.0129^{* * *} \\
{[0.0005]}\end{array}$ & $\begin{array}{l}0.0108^{* * *} \\
{[0.0006]}\end{array}$ \\
\hline $\mathrm{OP}$ & $\begin{array}{l}-0.0122^{* * *} \\
{[0.0012]}\end{array}$ & $\begin{array}{l}-0.0113^{* * *} \\
{[0.0012]}\end{array}$ & $\begin{array}{l}-0.0114^{* * *} \\
{[0.0008]}\end{array}$ & $\begin{array}{l}-0.0092^{* * *} \\
{[0.0009]}\end{array}$ & $\begin{array}{l}-0.0173^{* * *} \\
{[0.0005]}\end{array}$ & $\begin{array}{l}-0.0132^{* * *} \\
{[0.0013]}\end{array}$ & $\begin{array}{l}-0.0084^{* * *} \\
{[0.0012]}\end{array}$ & $\begin{array}{l}-0.0127^{* * *} \\
{[0.0011]}\end{array}$ & $\begin{array}{l}-0.0129^{* * *} \\
{[0.0008]}\end{array}$ \\
\hline OFD & $\begin{array}{l}\mathbf{0 . 0 0 6 4} \\
{[0.0166]}\end{array}$ & & & & & & & & \\
\hline FI & & $\begin{array}{l}-0.0315^{*} \\
{[0.0182]}\end{array}$ & & & & & & & \\
\hline FID & & & $\begin{array}{l}-0.0243^{* * *} \\
{[0.0067]}\end{array}$ & & & & & & \\
\hline FIA & & & & $\begin{array}{l}-\mathbf{0 . 0 5 3 4} \\
{[0.0106}\end{array}$ & & & & & \\
\hline FIE & & & & & $\begin{array}{l}\mathbf{0 . 1 4 3 9 * * *} \\
{[0.0102]}\end{array}$ & & & & \\
\hline FM & & & & & & $\begin{array}{l}\mathbf{0 . 0 6 7 9 * * *} \\
{[0.0090]}\end{array}$ & & & \\
\hline FMD & & & & & & & $\begin{array}{l}-0.0383^{* * *} \\
{[0.0086]}\end{array}$ & & \\
\hline FMA & & & & & & & & $\begin{array}{l}-\mathbf{0 . 0 0 3 3} \\
{[0.0008]}\end{array}$ & \\
\hline FME & & & & & & & & & $\begin{array}{l}\mathbf{0 . 0 3 7 0} \text { *** } \\
{[0.0046]}\end{array}$ \\
\hline Constant & $\begin{array}{l}0.1267^{* * *} \\
{[0.0139]}\end{array}$ & $\begin{array}{l}0.0853^{* * *} \\
{[0.0170]}\end{array}$ & $\begin{array}{l}0.0881^{* * *} \\
{[0.0064]}\end{array}$ & $\begin{array}{l}0.0546^{* * *} \\
{[0.0128]}\end{array}$ & $\begin{array}{l}0.1381^{* * *} \\
{[0.0054]}\end{array}$ & $\begin{array}{l}0.1853^{* * *} \\
{[0.0067]}\end{array}$ & $\begin{array}{l}0.0703^{* * *} \\
{[0.0097]}\end{array}$ & $\begin{array}{l}0.1264^{* * *} \\
{[0.0053]}\end{array}$ & $\begin{array}{l}0.1393^{* * *} \\
{[0.0058]}\end{array}$ \\
\hline Observations & 1,296 & 1,296 & 1,296 & 1,296 & 1,296 & 1,296 & 1,296 & 1,296 & 1,296 \\
\hline No of country & 81 & 81 & 81 & 81 & 81 & 81 & 81 & 81 & 81 \\
\hline No of IVs & 77 & 77 & 79 & 79 & 79 & 79 & 74 & 80 & 79 \\
\hline $\mathrm{AR}(2)$ test (p-value) & 0.590 & 0.590 & 0.596 & 0.574 & 0.567 & 0.565 & 0.610 & 0.592 & 0.580 \\
\hline Hansen test (p-value) & 0.204 & 0.283 & 0.232 & 0.379 & 0.233 & 0.250 & 0.465 & 0.289 & 0.219 \\
\hline
\end{tabular}

Note: two-step system GMM estimators; standard errors are in []; *, **, *** are significant levels at 10\%, 5\%, and 1\%, respectively. 
Table 8. Financial development and Consumption energy intensity: long-term effects

\begin{tabular}{|c|c|c|c|c|c|c|c|c|c|}
\hline Dep. var: CEI (CCR) & $(1)$ & $(2)$ & (3) & (4) & (5) & (6) & $(7)$ & (8) & (9) \\
\hline Income & $\begin{array}{l}2.2806^{* * *} \\
{[0.4094]}\end{array}$ & $\begin{array}{l}1.2183^{* * *} \\
{[0.2788]}\end{array}$ & $\begin{array}{l}1.9807^{* * *} \\
{[0.3649]}\end{array}$ & $\begin{array}{l}-0.3234 \\
{[0.3449]}\end{array}$ & $\begin{array}{l}2.1206^{* * *} \\
{[0.2555]}\end{array}$ & $\begin{array}{l}2.7248^{* * *} \\
{[0.4368]}\end{array}$ & $\begin{array}{l}2.7291^{* * *} \\
{[0.5672]}\end{array}$ & $\begin{array}{l}3.0308^{* * *} \\
{[0.4719]}\end{array}$ & $\begin{array}{l}2.6910^{* * *} \\
{[0.4041]}\end{array}$ \\
\hline Urban & $\begin{array}{l}-0.0561^{*} \\
{[0.0294]}\end{array}$ & $\begin{array}{l}-0.0066 \\
{[0.0195]}\end{array}$ & $\begin{array}{l}-0.0443^{* *} \\
{[0.0219]}\end{array}$ & $\begin{array}{l}-0.0041 \\
{[0.0372]}\end{array}$ & $\begin{array}{l}-0.0437^{* *} \\
{[0.0181]}\end{array}$ & $\begin{array}{l}-0.0881^{* * *} \\
{[0.0318]}\end{array}$ & $\begin{array}{l}-0.0867^{* *} \\
{[0.0384]}\end{array}$ & $\begin{array}{l}-0.1114^{* * *} \\
{[0.0354]}\end{array}$ & $\begin{array}{l}-0.0860^{* * *} \\
{[0.0297]}\end{array}$ \\
\hline Trade & $\begin{array}{l}-0.0029^{* * *} \\
{[0.0008]}\end{array}$ & $\begin{array}{l}-0.0027^{* * *} \\
{[0.0008]}\end{array}$ & $\begin{array}{l}-0.0026^{* * *} \\
{[0.0007]}\end{array}$ & $\begin{array}{l}0.0003 \\
{[0.0011]}\end{array}$ & $\begin{array}{l}-0.0034^{* * *} \\
{[0.0009]}\end{array}$ & $\begin{array}{l}-0.0031^{* * *} \\
{[0.0008]}\end{array}$ & $\begin{array}{l}-0.0032^{* * *} \\
{[0.0008]}\end{array}$ & $\begin{array}{l}-0.0033^{* * *} \\
{[0.0008]}\end{array}$ & $\begin{array}{l}-0.0030^{* * *} \\
{[0.0009]}\end{array}$ \\
\hline ES & $\begin{array}{l}0.1725^{* *} \\
{[0.0693]}\end{array}$ & $\begin{array}{l}0.3425^{* * *} \\
{[0.0680]}\end{array}$ & $\begin{array}{l}0.1067 \\
{[0.0667]}\end{array}$ & $\begin{array}{l}0.9095^{* * *} \\
{[0.0554]}\end{array}$ & $\begin{array}{l}0.2528^{* * *} \\
{[0.0891]}\end{array}$ & $\begin{array}{l}0.1131 \\
{[0.0721]}\end{array}$ & $\begin{array}{l}0.1033 \\
{[0.0851]}\end{array}$ & $\begin{array}{l}0.0774 \\
{[0.0746]}\end{array}$ & $\begin{array}{l}0.1196^{*} \\
{[0.0703]}\end{array}$ \\
\hline $\mathrm{OP}$ & $\begin{array}{l}-0.0113 \\
{[0.0325]}\end{array}$ & $\begin{array}{l}-0.0122 \\
{[0.0307]}\end{array}$ & $\begin{array}{l}-0.0093 \\
{[0.0347]}\end{array}$ & $\begin{array}{l}0.0763^{* * *} \\
{[0.0203]}\end{array}$ & $\begin{array}{l}-0.0201 \\
{[0.0333]}\end{array}$ & $\begin{array}{l}-0.0069 \\
{[0.0326]}\end{array}$ & $\begin{array}{l}-0.0023 \\
{[0.0349]}\end{array}$ & $\begin{array}{l}0.0051 \\
{[0.0338]}\end{array}$ & $\begin{array}{l}-0.0087 \\
{[0.0320]}\end{array}$ \\
\hline OFD & $\begin{array}{l}\mathbf{0 . 0 6 8 6} \\
{[0.1048]}\end{array}$ & & & & & & & & \\
\hline FI & & $\begin{array}{l}1.8421 * * * \\
{[0.2637]}\end{array}$ & & & & & & & \\
\hline FID & & & $\begin{array}{l}\mathbf{0 . 7 8 3 5 * * *} \\
{[0.2651]}\end{array}$ & & & & & & \\
\hline FIA & & & & $\begin{array}{l}4.8397^{* * *} \\
{[0.5446]}\end{array}$ & & & & & \\
\hline FIE & & & & & $\begin{array}{l}\mathbf{0 . 4 3 9 3}{ }^{* * *} \\
{[0.1604]}\end{array}$ & & & & \\
\hline FM & & & & & & $\begin{array}{l}-\mathbf{0 . 0 3 3 8} \\
{[0.0628]}\end{array}$ & & & \\
\hline FMD & & & & & & & $\begin{array}{l}-\mathbf{0 . 0 5 8 8} \\
{[0.1492]}\end{array}$ & & \\
\hline FMA & & & & & & & & $\begin{array}{l}-2.0022 \\
{[1.5100]}\end{array}$ & \\
\hline FME & & & & & & & & & $\begin{array}{l}-\mathbf{0 . 0 1 1 2} \\
{[0.0223]}\end{array}$ \\
\hline Constant & $\begin{array}{l}-9.0021^{* * *} \\
{[1.8346]} \\
\end{array}$ & $\begin{array}{l}-4.6842^{* * *} \\
{[1.3755]} \\
\end{array}$ & $\begin{array}{l}-7.0107^{* * *} \\
{[1.8268]} \\
\end{array}$ & $\begin{array}{l}1.9357 \\
{[1.4607]} \\
\end{array}$ & $\begin{array}{l}-8.9666^{* * *} \\
{[1.2162]} \\
\end{array}$ & $\begin{array}{l}-10.792^{* * *} \\
{[1.9423]} \\
\end{array}$ & $\begin{array}{l}-10.808^{* * *} \\
{[2.5004]} \\
\end{array}$ & $\begin{array}{l}-12.057^{* * *} \\
{[2.1190]} \\
\end{array}$ & $\begin{array}{l}-10.655^{* * *} \\
{[1.8031]} \\
\end{array}$ \\
\hline Observations & 16 & 16 & 16 & 16 & 16 & 16 & 16 & 16 & 16 \\
\hline R-squared & 0.9919 & 0.9933 & 0.9919 & 0.9957 & 0.9916 & 0.9916 & 0.9918 & 0.9909 & 0.9915 \\
\hline
\end{tabular}

Note: Canonical correlation regression (CCR) estimators; standard errors are in []; ${ }^{* *},{ }^{* * *}$ are significant levels at 10\%, 5\%, and $1 \%$, respectively. 
The role of FD in transforming energy intensity in consumption under the pressure of higher energy price is examined as well. The results of interaction terms between each financial indicator and oil price are presented in Table 9, the predictive margins analysis for the impacts of oil price on (depth, access, and efficiency) by two levels of FD are illustrated in figure 2. The results in Table 9 show the negative impacts of oil price on (depth, access, and efficiency), while the impacts of FD are properly consistent with findings in Table 7. That is, the financial institutions' depth, financial markets depth, and financial markets access have a negative impact, while the financial markets efficiency has a positive impact. The interaction terms between FD and oil price have a significant negative impact in case of financial institutions depth, financial institutions access, while they have a significant positive impact in case of financial institutions efficiency, financial markets depth, and financial markets access. This implies that the development of financial institutions is likely to help to induce a stronger negative impact of oil price on consumption energy intensity, while the development of financial markets has opposite effects. Figure 2 illustrates a clearer picture of the catalyst role of FD in transforming consumption energy intensity under higher energy price. Figure 2 shows that the slopes of the impacts of oil prices are steeper in case of higher financial markets depth and financial markets access, while it is less steep in the case of financial institutions access and financial institutions efficiency. 
Table 9. Financial development and Consumption energy intensity: the absorbing capability with the increases in energy price

\begin{tabular}{|c|c|c|c|c|c|c|c|c|c|}
\hline Dep. var: CEI (GMM) & $(1)$ & $(2)$ & $(3)$ & $(4)$ & (5) & $(6)$ & (7) & $(8)$ & (9) \\
\hline Indep. Var: FIN & OFD & $\mathrm{FI}$ & FID & FIA & FIE & FM & FMD & FMA & FME \\
\hline L.CEI & $\begin{array}{l}0.9568^{* * *} \\
{[0.0027]}\end{array}$ & $\begin{array}{l}0.9493^{* * *} \\
{[0.0027]}\end{array}$ & $\begin{array}{l}0.9666^{* * *} \\
{[0.0040]}\end{array}$ & $\begin{array}{l}0.9680^{* * *} \\
{[0.0031]}\end{array}$ & $\begin{array}{l}0.9483^{* * *} \\
{[0.0025]}\end{array}$ & $\begin{array}{l}0.9573^{* * *} \\
{[0.0028]}\end{array}$ & $\begin{array}{l}0.9781^{* * *} \\
{[0.0026]}\end{array}$ & $\begin{array}{l}0.9647^{* * *} \\
{[0.0033]}\end{array}$ & $\begin{array}{l}0.9515^{* * *} \\
{[0.0031]}\end{array}$ \\
\hline Income & $\begin{array}{l}0.0019 \\
{[0.0024]}\end{array}$ & $\begin{array}{l}0.0346^{* * *} \\
{[0.0036]}\end{array}$ & $\begin{array}{l}0.0133^{* * *} \\
{[0.0033]}\end{array}$ & $\begin{array}{l}0.0145^{* * *} \\
{[0.0024]}\end{array}$ & $\begin{array}{l}0.0040^{* *} \\
{[0.0020]}\end{array}$ & $\begin{array}{l}0.0024 \\
{[0.0025]}\end{array}$ & $\begin{array}{l}-0.0012 \\
{[0.0026]}\end{array}$ & $\begin{array}{l}0.0080^{* * *} \\
{[0.0020]}\end{array}$ & $\begin{array}{l}0.0134^{* * *} \\
{[0.0021]}\end{array}$ \\
\hline Urban & $\begin{array}{l}0.0006^{* * *} \\
{[0.0001]}\end{array}$ & $\begin{array}{l}0.0002^{* *} \\
{[0.0001]}\end{array}$ & $\begin{array}{l}0.0003^{* * *} \\
{[0.0001]}\end{array}$ & $\begin{array}{l}0.0002^{*} \\
{[0.0001]}\end{array}$ & $\begin{array}{l}0.0009^{* * *} \\
{[0.0001]}\end{array}$ & $\begin{array}{l}0.0006^{* * *} \\
{[0.0001]}\end{array}$ & $\begin{array}{l}0.0001^{* *} \\
{[0.0001]}\end{array}$ & $\begin{array}{l}0.0002^{* *} \\
{[0.0001]}\end{array}$ & $\begin{array}{l}0.0006^{* * *} \\
{[0.0001]}\end{array}$ \\
\hline Trade & $\begin{array}{l}0.0004^{* * *} \\
{[0.00005]}\end{array}$ & $\begin{array}{l}0.0004^{* * *} \\
{[0.00004]}\end{array}$ & $\begin{array}{l}0.0003^{* * *} \\
{[0.00004]}\end{array}$ & $\begin{array}{l}0.0002^{* * *} \\
{[0.00003]}\end{array}$ & $\begin{array}{l}0.0003^{* * *} \\
{[0.00004]}\end{array}$ & $\begin{array}{l}0.0004^{* * *} \\
{[0.00003]}\end{array}$ & $\begin{array}{l}0.0002^{* * *} \\
{[0.00003]}\end{array}$ & $\begin{array}{l}0.0003^{* * *} \\
{[0.00003]}\end{array}$ & $\begin{array}{l}0.0004^{* * *} \\
{[0.00004]}\end{array}$ \\
\hline ES & $\begin{array}{l}0.0130^{* * *} \\
{[0.0011]}\end{array}$ & $\begin{array}{l}0.0132^{* * *} \\
{[0.0009]}\end{array}$ & $\begin{array}{l}0.0088^{* * *} \\
{[0.0007]}\end{array}$ & $\begin{array}{l}0.0107^{* * *} \\
{[0.0008]}\end{array}$ & $\begin{array}{l}0.0143^{* * *} \\
{[0.0006]}\end{array}$ & $\begin{array}{l}0.0132^{* * *} \\
{[0.0009]}\end{array}$ & $\begin{array}{l}0.0093^{* * *} \\
{[0.0007]}\end{array}$ & $\begin{array}{l}0.0138^{* * *} \\
{[0.0005]}\end{array}$ & $\begin{array}{l}0.0107^{* * *} \\
{[0.0006]}\end{array}$ \\
\hline OP & $\begin{array}{l}-\mathbf{0 . 0 1 4 1} * * * \\
{[0.0020]}\end{array}$ & $\begin{array}{l}-\mathbf{0 . 0 0 8 3} * * * \\
{[0.0015]}\end{array}$ & $\begin{array}{l}-\mathbf{0 . 0 1 0 5} * * * \\
{[0.0014]}\end{array}$ & $\begin{array}{l}-\mathbf{0 . 0 0 5 9} * * * \\
{[0.0014]}\end{array}$ & $\begin{array}{l}-\mathbf{0 . 0 6 5 1 * * *} \\
{[0.0068]}\end{array}$ & $\begin{array}{l}-\mathbf{0 . 0 1 5 2 * * *} \\
{[0.0016]}\end{array}$ & $\begin{array}{l}-\mathbf{0 . 0 1 3 8 * *} \\
{[0.0016]}\end{array}$ & $\begin{array}{l}-\mathbf{0 . 0 1 7 1} * * * \\
{[0.0016]}\end{array}$ & $\begin{array}{l}-\mathbf{0 . 0 1 1 8} * * * \\
{[0.0017]}\end{array}$ \\
\hline FIN & $\begin{array}{l}\mathbf{0 . 0 6 6 2}{ }^{* * *} \\
{[0.0234]}\end{array}$ & $\begin{array}{l}-0.0670^{* *} \\
{[0.0270]}\end{array}$ & $\begin{array}{l}-\mathbf{0 . 0 0 7 9} \\
{[0.0179]}\end{array}$ & $\begin{array}{l}\mathbf{0 . 0 0 7 8} \\
{[0.0151]}\end{array}$ & $\begin{array}{l}-\mathbf{0 . 1 3 1 4} * * * \\
{[0.0332]}\end{array}$ & $\begin{array}{l}\mathbf{0 . 0 2 8 2} \\
{[0.0196]}\end{array}$ & $\begin{array}{l}-\mathbf{0 . 0 4 6 1 * *} \\
{[0.0186]}\end{array}$ & $\begin{array}{l}-\mathbf{0 . 0 7 1 4} * * * \\
{[0.0128]}\end{array}$ & $\begin{array}{l}\mathbf{0 . 0 4 3 4 * * *} \\
{[0.0116]}\end{array}$ \\
\hline OP*FIN & $\begin{array}{l}\mathbf{0 . 0 0 0 1} \\
{[0.0039]}\end{array}$ & $\begin{array}{l}-\mathbf{0 . 0 0 6 7 * *} \\
{[0.0026]}\end{array}$ & $\begin{array}{l}-\mathbf{0 . 0 0 2 8} \\
{[0.0024]}\end{array}$ & $\begin{array}{l}-\mathbf{0 . 0 1 1 9 * * *} \\
{[0.0026]}\end{array}$ & $\begin{array}{l}\mathbf{0 . 0 7 3 6 * * *} \\
{[0.0094]}\end{array}$ & $\begin{array}{l}\mathbf{0 . 0 0 8 0} \text { ** } \\
{[0.0035]}\end{array}$ & $\begin{array}{l}\mathbf{0 . 0 1 5 5 * * *} \\
{[0.0033]}\end{array}$ & $\begin{array}{l}\mathbf{0 . 0 1 6 4 * * *} \\
{[0.0027]}\end{array}$ & $\begin{array}{l}-\mathbf{0 . 0 0 1 6} \\
{[0.0025]}\end{array}$ \\
\hline Constant & $\begin{array}{l}0.1846^{* * *} \\
{[0.0114]}\end{array}$ & $\begin{array}{l}0.0230 \\
{[0.0151]}\end{array}$ & $\begin{array}{l}0.0896^{* * *} \\
{[0.0065]}\end{array}$ & $\begin{array}{l}0.0564^{* * *} \\
{[0.0149]}\end{array}$ & $\begin{array}{l}0.3269^{* * *} \\
{[0.0269]}\end{array}$ & $\begin{array}{l}0.1880^{* * *} \\
{[0.0071]}\end{array}$ & $\begin{array}{l}0.1473^{* * *} \\
{[0.0079]}\end{array}$ & $\begin{array}{l}0.1418^{* * *} \\
{[0.0058]}\end{array}$ & $\begin{array}{l}0.1347^{* * *} \\
{[0.0082]}\end{array}$ \\
\hline Observations & 1,296 & 1,296 & 1,296 & 1,296 & 1,296 & 1,296 & 1,296 & 1,296 & 1,296 \\
\hline No of country & 81 & 81 & 81 & 81 & 81 & 81 & 81 & 81 & 81 \\
\hline No of IVs & 79 & 79 & 79 & 79 & 79 & 79 & 77 & 80 & 79 \\
\hline$A R(2)$ test (p-value) & 0.574 & 0.593 & 0.595 & 0.584 & 0.539 & 0.565 & 0.572 & 0.592 & 0.578 \\
\hline Hansen test (p-value) & 0.346 & 0.447 & 0.208 & 0.341 & 0.209 & 0.239 & 0.198 & 0.255 & 0.218 \\
\hline
\end{tabular}

Note: two-step system GMM estimators; standard errors are in []; *, **, *** are significant levels at 10\%, 5\%, and 1\%, respectively. 


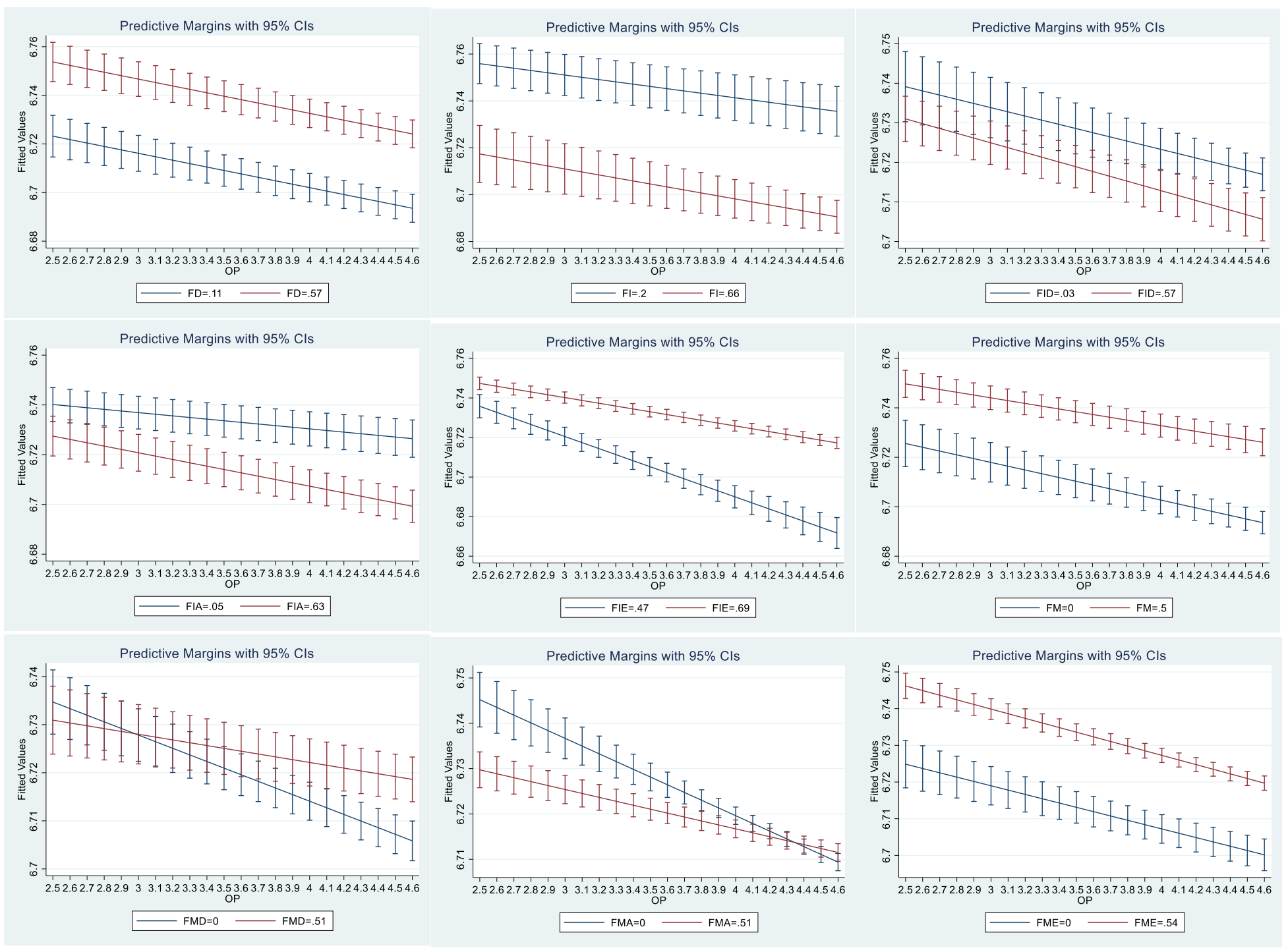

Figure 2. Predictive margins of the effects of oil price on Consumption Energy intensity by levels of financial development 
Table 10. Financial development and Consumption energy intensity: three subsamples

\begin{tabular}{|c|c|c|c|c|c|c|c|c|c|}
\hline LMEs & & & & & & & & & \\
\hline Dep. var: CEI (FGLS) & (1) & (2) & (3) & (4) & (5) & (6) & (7) & (8) & (9) \\
\hline Indep. Var: FIN & OFD & FI & FID & FIA & FIE & FM & FMD & FMA & FME \\
\hline Income(-1) & $\begin{array}{l}0.3585^{* * *} \\
{[0.0617]}\end{array}$ & $\begin{array}{l}0.3962^{* * *} \\
{[0.0634]}\end{array}$ & $\begin{array}{l}0.5609^{* * *} \\
{[0.0636]}\end{array}$ & $\begin{array}{l}0.4880^{* * *} \\
{[0.0503]}\end{array}$ & $\begin{array}{l}0.6986^{* * *} \\
{[0.0616]}\end{array}$ & $\begin{array}{l}0.4818^{* * *} \\
{[0.0591]}\end{array}$ & $\begin{array}{l}0.5883^{* * *} \\
{[0.0601]}\end{array}$ & $\begin{array}{l}0.5925^{* * *} \\
{[0.0710]}\end{array}$ & $\begin{array}{l}0.5815^{* * *} \\
{[0.0538]}\end{array}$ \\
\hline $\operatorname{Urban}(-1)$ & $\begin{array}{l}0.0224^{* * *} \\
{[0.0024]}\end{array}$ & $\begin{array}{l}0.0173^{* * *} \\
{[0.0023]}\end{array}$ & $\begin{array}{l}0.0148^{* * *} \\
{[0.0024]}\end{array}$ & $\begin{array}{l}0.0117^{* * *} \\
{[0.0021]}\end{array}$ & $\begin{array}{l}0.0124^{* * *} \\
{[0.0025]}\end{array}$ & $\begin{array}{l}0.0209^{* * *} \\
{[0.0025]}\end{array}$ & $\begin{array}{l}0.0162^{* * *} \\
{[0.0025]}\end{array}$ & $\begin{array}{l}0.0153^{* * *} \\
{[0.0026]}\end{array}$ & $\begin{array}{l}0.0195^{* * *} \\
{[0.0024]}\end{array}$ \\
\hline Trade $(-1)$ & $\begin{array}{l}0.0089^{* * *} \\
{[0.0008]}\end{array}$ & $\begin{array}{l}0.0082^{* * *} \\
{[0.0009]}\end{array}$ & $\begin{array}{l}0.0088^{* * *} \\
{[0.0009]}\end{array}$ & $\begin{array}{l}0.0073^{* * *} \\
{[0.0008]}\end{array}$ & $\begin{array}{l}0.0086^{* * *} \\
{[0.0009]}\end{array}$ & $\begin{array}{l}0.0091^{* * *} \\
{[0.0009]}\end{array}$ & $\begin{array}{l}0.0093^{* * *} \\
{[0.0009]}\end{array}$ & $\begin{array}{l}0.0090^{* * *} \\
{[0.0009]}\end{array}$ & $\begin{array}{l}0.0079^{* * *} \\
{[0.0009]}\end{array}$ \\
\hline $\mathrm{ES}(-1)$ & $\begin{array}{l}0.2288^{* * *} \\
{[0.0144]}\end{array}$ & $\begin{array}{l}0.2564^{* * *} \\
{[0.0139]}\end{array}$ & $\begin{array}{l}0.2785^{* * *} \\
{[0.0142]}\end{array}$ & $\begin{array}{l}0.2394^{* * *} \\
{[0.0127]}\end{array}$ & $\begin{array}{l}0.2847^{* * *} \\
{[0.0143]}\end{array}$ & $\begin{array}{l}0.2399 * * \\
{[0.0148]}\end{array}$ & $\begin{array}{l}0.2666^{* * *} \\
{[0.0150]}\end{array}$ & $\begin{array}{l}0.2817^{* * *} \\
{[0.0143]}\end{array}$ & $\begin{array}{l}0.2321^{* * *} \\
{[0.0147]}\end{array}$ \\
\hline $\mathrm{OP}$ & $\begin{array}{l}-0.2303^{* * *} \\
{[0.0410]}\end{array}$ & $\begin{array}{l}-0.2794^{* * *} \\
{[0.0424]}\end{array}$ & $\begin{array}{l}-0.2498^{* * *} \\
{[0.0445]}\end{array}$ & $\begin{array}{l}-0.2993^{* * *} \\
{[0.0385]}\end{array}$ & $\begin{array}{l}-0.2242^{* * *} \\
{[0.0442]}\end{array}$ & $\begin{array}{l}-0.2021^{* * *} \\
{[0.0422]}\end{array}$ & $\begin{array}{l}-0.2442^{* * *} \\
{[0.0442]}\end{array}$ & $\begin{array}{l}-0.2194^{* * *} \\
{[0.0444]}\end{array}$ & $\begin{array}{l}-0.1827^{* * *} \\
{[0.0418]}\end{array}$ \\
\hline FIN(-1) & $\begin{array}{l}\mathbf{2 . 9 1 3 1} * * * \\
{[0.3167]}\end{array}$ & $\begin{array}{l}\mathbf{2 . 9 2 9 9 * * *} \\
{[0.3790]}\end{array}$ & $\begin{array}{l}\mathbf{1 . 3 4 8 7} * * * \\
{[0.4132]}\end{array}$ & $\begin{array}{l}2.8706^{* * *} \\
{[0.2167]}\end{array}$ & $\begin{array}{l}-\mathbf{0 . 3 0 4 5} \\
{[0.1904]}\end{array}$ & $\begin{array}{l}1.5505^{* * *} \\
{[0.2129]}\end{array}$ & $\begin{array}{l}\mathbf{0 . 9 6 0 7 * * *} \\
{[0.2940]}\end{array}$ & $\begin{array}{l}\mathbf{0 . 3 9 1 5} \\
{[0.2516]}\end{array}$ & $\begin{array}{l}\mathbf{0 . 7 5 4 0}^{* * *} \\
{[0.0898]}\end{array}$ \\
\hline Constant & $\begin{array}{l}0.9247^{* * *} \\
{[0.3446]} \\
\end{array}$ & $\begin{array}{l}0.7632^{* *} \\
{[0.3548]}\end{array}$ & $\begin{array}{l}0.0685 \\
{[0.3756]} \\
\end{array}$ & $\begin{array}{l}0.9490^{* * *} \\
{[0.3058]}\end{array}$ & $\begin{array}{l}-0.6009^{*} \\
{[0.3333]}\end{array}$ & $\begin{array}{l}0.3174 \\
{[0.3357]}\end{array}$ & $\begin{array}{l}-0.1100 \\
{[0.3521]}\end{array}$ & $\begin{array}{l}-0.2008 \\
{[0.3925]}\end{array}$ & $\begin{array}{l}-0.2590 \\
{[0.3116]}\end{array}$ \\
\hline Observations & 496 & 496 & 496 & 496 & 496 & 496 & 496 & 496 & 496 \\
\hline No of Country & 31 & 31 & 31 & 31 & 31 & 31 & 31 & 31 & 31 \\
\hline \multicolumn{10}{|l|}{ UMEs } \\
\hline Dep.var: CEI (FGLS) & $(1)$ & (2) & (3) & (4) & (5) & (6) & (7) & $(8)$ & (9) \\
\hline Indep. Var: FIN & OFD & FI & FID & FIA & FIE & FM & FMD & FMA & FME \\
\hline Income(-1) & $\begin{array}{l}0.2343^{* * *} \\
{[0.0685]}\end{array}$ & $\begin{array}{l}0.2737^{* * *} \\
{[0.0697]}\end{array}$ & $\begin{array}{l}0.2593^{* * *} \\
{[0.0692]}\end{array}$ & $\begin{array}{l}0.2732^{* * *} \\
{[0.0696]}\end{array}$ & $\begin{array}{l}0.2449^{* * *} \\
{[0.0664]}\end{array}$ & $\begin{array}{l}0.2291^{* * *} \\
{[0.0668]}\end{array}$ & $\begin{array}{l}0.2131^{* * *} \\
{[0.0665]}\end{array}$ & $\begin{array}{l}0.2666^{* * *} \\
{[0.0654]}\end{array}$ & $\begin{array}{l}0.2169^{* * *} \\
{[0.0656]}\end{array}$ \\
\hline $\operatorname{Urban}(-1)$ & $\begin{array}{l}0.0079^{* * *} \\
{[0.0018]}\end{array}$ & $\begin{array}{l}0.0074^{* * *} \\
{[0.0018]}\end{array}$ & $\begin{array}{l}0.0075^{* * *} \\
{[0.0019]}\end{array}$ & $\begin{array}{l}0.0081^{* * *} \\
{[0.0018]}\end{array}$ & $\begin{array}{l}0.0076^{* * *} \\
{[0.0018]}\end{array}$ & $\begin{array}{l}0.0075^{* * *} \\
{[0.0018]}\end{array}$ & $\begin{array}{l}0.0074^{* * *} \\
{[0.0018]}\end{array}$ & $\begin{array}{l}0.0104^{* * *} \\
{[0.0019]}\end{array}$ & $\begin{array}{l}0.0084^{* * *} \\
{[0.0018]}\end{array}$ \\
\hline Trade $(-1)$ & $\begin{array}{l}0.0049^{* * *} \\
{[0.0006]}\end{array}$ & $\begin{array}{l}0.0052^{* * *} \\
{[0.0006]}\end{array}$ & $\begin{array}{l}0.0051^{* * *} \\
{[0.0006]}\end{array}$ & $\begin{array}{l}0.0050^{* * *} \\
{[0.0006]}\end{array}$ & $\begin{array}{l}0.0051^{* * *} \\
{[0.0006]}\end{array}$ & $\begin{array}{l}0.0049^{* * *} \\
{[0.0006]}\end{array}$ & $\begin{array}{l}0.0044^{* * *} \\
{[0.0006]}\end{array}$ & $\begin{array}{l}0.0051^{* * *} \\
{[0.0006]}\end{array}$ & $\begin{array}{l}0.0053^{* * *} \\
{[0.0006]}\end{array}$ \\
\hline $\mathrm{ES}(-1)$ & $\begin{array}{l}0.1593^{* * *} \\
{[0.0170]}\end{array}$ & $\begin{array}{l}0.1661^{* * *} \\
{[0.0166]}\end{array}$ & $\begin{array}{l}0.1640^{* * *} \\
{[0.0165]}\end{array}$ & $\begin{array}{l}0.1638^{* * *} \\
{[0.0164]}\end{array}$ & $\begin{array}{l}0.1648^{* * *} \\
{[0.0167]}\end{array}$ & $\begin{array}{l}0.1548^{* * *} \\
{[0.0169]}\end{array}$ & $\begin{array}{l}0.1508^{* * *} \\
{[0.0167]}\end{array}$ & $\begin{array}{l}0.1752^{* * *} \\
{[0.0165]}\end{array}$ & $\begin{array}{l}0.1502^{* * *} \\
{[0.0165]}\end{array}$ \\
\hline $\mathrm{OP}$ & $\begin{array}{l}-0.0284 \\
{[0.0426]}\end{array}$ & $\begin{array}{l}-0.0136 \\
{[0.0435]}\end{array}$ & $\begin{array}{l}-0.0240 \\
{[0.0425]}\end{array}$ & $\begin{array}{l}-0.0160 \\
{[0.0431]}\end{array}$ & $\begin{array}{l}-0.0175 \\
{[0.0442]}\end{array}$ & $\begin{array}{l}-0.0237 \\
{[0.0423]}\end{array}$ & $\begin{array}{l}-0.0344 \\
{[0.0420]}\end{array}$ & $\begin{array}{l}-0.0334 \\
{[0.0418]}\end{array}$ & $\begin{array}{l}-0.0158 \\
{[0.0418]}\end{array}$ \\
\hline FIN(-1) & $\begin{array}{l}\mathbf{0 . 1 2 8 6} \\
{[0.1646]}\end{array}$ & $\begin{array}{l}-0.2150 \\
{[0.1829]}\end{array}$ & $\begin{array}{l}-0.0625 \\
{[0.1107]}\end{array}$ & $\begin{array}{l}-0.1649 \\
{[0.1422]}\end{array}$ & $\begin{array}{l}-0.0990 \\
{[0.1531]}\end{array}$ & $\begin{array}{l}\mathbf{0 . 2 0 7 4}^{*} \\
{[0.1141]}\end{array}$ & $\begin{array}{l}\mathbf{0 . 3 3 9 8}{ }^{* * *} \\
{[0.1143]}\end{array}$ & $\begin{array}{l}-0.4076^{* * *} \\
{[0.1219]}\end{array}$ & $\begin{array}{l}\mathbf{0 . 2 4 9 6 * * *} \\
{[0.0701]}\end{array}$ \\
\hline Constant & $\begin{array}{l}3.3556^{* * *} \\
{[0.4900]} \\
\end{array}$ & $\begin{array}{l}3.0447^{* * *} \\
{[0.4943]} \\
\end{array}$ & $\begin{array}{l}3.1572^{* * *} \\
{[0.4884]} \\
\end{array}$ & $\begin{array}{l}3.0138^{* * *} \\
{[0.5046]} \\
\end{array}$ & $\begin{array}{l}3.2826^{* * *} \\
{[0.4716]} \\
\end{array}$ & $\begin{array}{l}3.4251^{* * *} \\
{[0.4759]} \\
\end{array}$ & $\begin{array}{l}3.6516^{* * *} \\
{[0.4814]} \\
\end{array}$ & $\begin{array}{l}2.9515^{* * *} \\
{[0.4673]} \\
\end{array}$ & $\begin{array}{l}3.4145^{* * *} \\
{[0.4610]} \\
\end{array}$ \\
\hline Observations & 336 & 336 & 336 & 336 & 336 & 336 & 336 & 336 & 336 \\
\hline No of Country & 21 & 21 & 21 & 21 & 21 & 21 & 21 & 21 & 21 \\
\hline \multicolumn{10}{|l|}{ HIEs } \\
\hline Dep. var: CEI (FGLS) & $(1)$ & (2) & $(3)$ & $(4)$ & $(5)$ & $(6)$ & (7) & $(8)$ & $(9)$ \\
\hline
\end{tabular}




\begin{tabular}{|c|c|c|c|c|c|c|c|c|c|}
\hline Indep. Var: FIN & FD & FI & FID & FIA & FIE & $\mathrm{FM}$ & FMD & FMA & FME \\
\hline Income(-1) & $\begin{array}{l}0.4428^{* * *} \\
{[0.0326]}\end{array}$ & $\begin{array}{l}0.5632 * * * \\
{[0.0326]}\end{array}$ & $\begin{array}{l}0.5989^{* * *} \\
{[0.0309]}\end{array}$ & $\begin{array}{l}0.4918^{* * *} \\
{[0.0218]}\end{array}$ & $\begin{array}{l}0.4399 * * * \\
{[0.0192]}\end{array}$ & $\begin{array}{l}0.4024^{* * *} \\
{[0.0253]}\end{array}$ & $\begin{array}{l}0.4400^{* * *} \\
{[0.0246]}\end{array}$ & $\begin{array}{l}0.4694^{* * *} \\
{[0.0195]}\end{array}$ & $\begin{array}{l}0.4105^{* * *} \\
{[0.0190]}\end{array}$ \\
\hline $\operatorname{Urban}(-1)$ & $\begin{array}{l}0.0011 \\
{[0.0009]}\end{array}$ & $\begin{array}{l}0.0001 \\
{[0.0009]}\end{array}$ & $\begin{array}{l}0.0008 \\
{[0.0008]}\end{array}$ & $\begin{array}{l}0.0004 \\
{[0.0009]}\end{array}$ & $\begin{array}{l}0.0011 \\
{[0.0009]}\end{array}$ & $\begin{array}{l}0.0010 \\
{[0.0008]}\end{array}$ & $\begin{array}{l}0.0009 \\
{[0.0009]}\end{array}$ & $\begin{array}{l}0.0009 \\
{[0.0009]}\end{array}$ & $\begin{array}{l}0.0007 \\
{[0.0008]}\end{array}$ \\
\hline Trade $(-1)$ & $\begin{array}{l}0.0006^{*} \\
{[0.0004]}\end{array}$ & $\begin{array}{l}0.0003 \\
{[0.0003]}\end{array}$ & $\begin{array}{l}0.0005 \\
{[0.0003]}\end{array}$ & $\begin{array}{l}0.0003 \\
{[0.0004]}\end{array}$ & $\begin{array}{l}0.0003 \\
{[0.0004]}\end{array}$ & $\begin{array}{l}0.0007^{* *} \\
{[0.0003]}\end{array}$ & $\begin{array}{l}0.0006^{*} \\
{[0.0003]}\end{array}$ & $\begin{array}{l}0.0005 \\
{[0.0003]}\end{array}$ & $\begin{array}{l}0.0008^{* *} \\
{[0.0003]}\end{array}$ \\
\hline $\mathrm{ES}(-1)$ & $\begin{array}{l}0.0989^{* * *} \\
{[0.0079]}\end{array}$ & $\begin{array}{l}0.0944^{* * *} \\
{[0.0079]}\end{array}$ & $\begin{array}{l}0.0970^{* * *} \\
{[0.0077]}\end{array}$ & $\begin{array}{l}0.0969^{* * *} \\
{[0.0080]}\end{array}$ & $\begin{array}{l}0.1013^{* * *} \\
{[0.0079]}\end{array}$ & $\begin{array}{l}0.0951^{* * *} \\
{[0.0080]}\end{array}$ & $\begin{array}{l}0.0979^{* * *} \\
{[0.0080]}\end{array}$ & $\begin{array}{l}0.1002^{* * *} \\
{[0.0081]}\end{array}$ & $\begin{array}{l}0.0978^{* * *} \\
{[0.0077]}\end{array}$ \\
\hline $\mathrm{OP}$ & $\begin{array}{l}-0.1006^{* * *} \\
{[0.0198]}\end{array}$ & $\begin{array}{l}-0.0862^{* * *} \\
{[0.0198]}\end{array}$ & $\begin{array}{l}-0.0998^{* * *} \\
{[0.0192]}\end{array}$ & $\begin{array}{l}-0.0907^{* * *} \\
{[0.0201]}\end{array}$ & $\begin{array}{l}-0.1038^{* * *} \\
{[0.0197]}\end{array}$ & $\begin{array}{l}-0.0985^{* * *} \\
{[0.0195]}\end{array}$ & $\begin{array}{l}-0.1024^{* * *} \\
{[0.0198]}\end{array}$ & $\begin{array}{l}-0.0999^{* * *} \\
{[0.0197]}\end{array}$ & $\begin{array}{l}-0.0891^{* * *} \\
{[0.0193]}\end{array}$ \\
\hline FIN(-1) & $\begin{array}{l}\mathbf{0 . 0 7 3 0} \\
{[0.1044]}\end{array}$ & $\begin{array}{l}-\mathbf{0 . 3 9 9 5 * * *} \\
{[0.1121]}\end{array}$ & $\begin{array}{l}-\mathbf{0 . 4 0 1 4} * * * \\
{[0.0776]}\end{array}$ & $\begin{array}{l}-\mathbf{0 . 1 2 3 2} * * \\
{[0.0601]}\end{array}$ & $\begin{array}{l}\mathbf{0 . 2 3 6 4 * *} \\
{[0.1032]}\end{array}$ & $\begin{array}{l}\mathbf{0 . 2 1 3 9} \\
{[0.0689]}\end{array}$ & $\begin{array}{l}\mathbf{0 . 0 6 9 9} \\
{[0.0569]}\end{array}$ & $\begin{array}{l}-\mathbf{0 . 0 3 0 2} \\
{[0.0444]}\end{array}$ & $\begin{array}{l}\mathbf{0 . 1 8 6 0 * * *} \\
{[0.0361]}\end{array}$ \\
\hline Constant & $\begin{array}{l}3.1749^{* * *} \\
{[0.2751]}\end{array}$ & $\begin{array}{l}2.3352^{* * *} \\
{[0.2560]}\end{array}$ & $\begin{array}{l}1.9011^{* * *} \\
{[0.2729]}\end{array}$ & $\begin{array}{l}2.8455^{* * *} \\
{[0.1905]}\end{array}$ & $\begin{array}{l}3.0897^{* * *} \\
{[0.1717]}\end{array}$ & $\begin{array}{l}3.5316^{* * *} \\
{[0.2348]}\end{array}$ & $\begin{array}{l}3.2365^{* * *} \\
{[0.2429]}\end{array}$ & $\begin{array}{l}2.9694^{* * *} \\
{[0.1879]}\end{array}$ & $\begin{array}{l}3.4197^{* * *} \\
{[0.1825]}\end{array}$ \\
\hline Observations & 464 & 464 & 464 & 464 & 464 & 464 & 464 & 464 & 464 \\
\hline No of Country & 29 & 29 & 29 & 29 & 29 & 29 & 29 & 29 & 29 \\
\hline
\end{tabular}

Note: Feasible generalized least squares (FGLS) estimators; standard errors are in []; *,**,** are significant levels at 10\%, 5\%, and 1\%, respectively. 
At last, the influences of FD on consumption energy intensity in three subsamples are presented in Table 10. In LMEs, the results show the positive impacts of most financial indicators on consumption energy intensity. In UMEs, the result shows negative impacts of financial institutions and its three dimensions, and financial markets access on consumption energy intensity, while financial institutions and its dimensions of depth and efficiency have a positive impact. In HIEs, financial institutions and its dimensions of depth and access, and financial markets access have a negative impact, while financial institutions efficiency, financial markets and its dimensions of depth and efficiency have positive impacts. Overall, the results show that the FD mostly induces higher consumption energy intensity in LMEs, which then have some difference in cases of UMEs and HIEs. In UMEs and HIEs, the financial institutions are likely to help to reduce consumption energy intensity, while financial markets cause higher consumption energy intensity.

In summary, the key findings and empirically consistent results show that the FD induces higher production energy intensity except in the case of financial institutions efficiency. These effects are consistently found and documented in the long run. In contrast, the financial depth and financial access have a negative impact on consumption energy intensity, while financial efficiency has a positive impact. In the long run, financial institutions appear to increase consumption energy intensity, while financial markets have opposite effects. Notably, the results show the important catalyst role of FD in transforming energy usage due to higher energy prices. The reduction of production energy intensity in the face of energy price shocks is supported by the higher level of FD. Interestingly, the reduction of consumption energy intensity in the context of higher energy price is supported by stronger financial institutions. The FD is found to decrease production energy intensity in HIEs but increase production energy intensity in UMEs and has mixed effects in LMEs. The FD is found to induce higher consumption energy intensity in LMEs, which is same for the impacts of financial markets in UMEs and HIEs. The financial institutions appear to reduce consumption energy intensity in UMEs and HIEs. These empirical findings have profound policy implications for policymakers in the context of the debate on the role of finance in energy efficiency and tackling economic and environmental challenges. 


\section{Conclusion and Policy Implications}

Contribution and significance of the financial sector for the real economy has been well recognized and established in the literature, and recently the focus has been extended to the role of financial developed in energy consumption and environment. In this context, our empirical results lead us to conclude that the FD induces higher production energy intensity except for the decreasing effect of financial institutions efficiency. These effects are documented consistently in the long run. It implies that the development of the financial sector could have a negative environmental impact due to increasing energy intensity except for the higher financial institutions efficiency. This result highlights a very interesting implication that the higher efficiency in financial institutions, i.e. banks, would help reduce energy intensity. This effect leads to infer that the higher efficiency, or the lower cost of financial fund and services, would help firms to advance the technology in production, which help reduce the energy intensity. Meanwhile, other financial indicators have increasing impacts on energy intensity implying the side effect of FD on the environment. The results suggest that in policy setting, the governments should have more financial projects aiming at providing low-cost fund for technology transformation and energy intensity reduction.

Our results also indicate that financial depth and financial access have a negative impact on consumption energy intensity. This is an interesting finding since it leads us to infer that the access and depth of financial sectors help to reduce energy intensity in consumption. Implying that the energy intensity in consumption is constrained by the available fund and size of fund for their transformation. As per previous results, only financial institutions efficiency has a reducing effect on production energy intensity, however, the reducing effects of financial access and financial depth from both financial institutions and financial markets on consumption energy intensity are also very significant. Therefore, in the policy context, it needs to develop financial sectors to ensure every citizen's financial access and to get the financial funds for energy transformation toward higher energy efficiency.

Notably, in the light of our results, we conclude on the important catalyzing role of FD in transforming the energy usage in the context of high energy price. The reduction in production energy intensity in the face of high energy price shocks is supported by a higher 
level of the FD. Interestingly, the reduction of consumption energy intensity due to the high energy prices is also supported by stronger financial institutions. This implies that the development of the financial sector, especially financial institutions, is vital for energy intensity transformation in the face of oil price shocks. In the policy setting, the long-term aims of higher energy intensity can be supported by green finance in the context of high oil price. This will help not only firms but also citizens in reducing their energy consumption intensity. Lastly, the results in countries at different income levels lead us to conclude that the FD decreases production energy intensity in HIEs but increase production energy intensity in UMEs and has mixed effects in LMEs. The FD is found to induce higher consumption energy intensity in LMEs, which is same for the impact of financial markets in UMEs and HIEs. Meanwhile, financial institutions appear to reduce consumption energy intensity in UMEs and HIEs. Overall, the results are a prima facie manifestation of the inefficiency in the strategy of financial development in LMEs and UMEs. In the policy setting, this would imply that a greener and inclusive development of the financial sector in LMEs and UMEs should be the prime focus of development efforts. 


\section{References}

Adams, S., Klobodu, E.K.M., 2018. Financial development and environmental degradation: Does political regime matter? J. Clean. Prod. 197, 1472-1479.

Al-Mulali, U., Ozturk, I., Lean, H.H., 2015. The influence of economic growth, urbanization, trade openness, financial development, and renewable energy on pollution in Europe. Nat Hazards 79, 621-644.

Al Mamun, M., Sohag, K., Shahbaz, M., Hammoudeh, S., 2018. Financial markets, innovations and cleaner energy production in OECD countries. Energy Econ. 72, 236-254.

Alam, A., Malik, I.A., Abdullah, A.B., Hassan, A., Faridullah, Awan, U., Ali, G., Zaman, K., Naseem, I., 2015. Does financial development contribute to SAARC'S energy demand? From energy crisis to energy reforms. Renew. Sust. Energ. Rev. 41, 818-829.

Ali, Q., Khan, M.T.I., Khan, M.N.I., 2018. Dynamics between financial development, tourism, sanitation, renewable energy, trade and total reserves in 19 Asia cooperation dialogue members. J. Clean. Prod. 179, 114-131.

Anderson, T.W., Hsiao, C., 1982. Formulation and estimation of dynamic models using panel data. Journal of econometrics $18,47-82$.

Arellano, M., Bond, S., 1991. Some tests of specification for panel data: Monte Carlo evidence and an application to employment equations. Rev. Econ. Stud. 58, 277-297.

Arellano, M., Bover, O., 1995. Another look at the instrumental variable estimation of error-components models. J. Econom. 68, 29-51.

Azam, M., Khan, A.Q., Zaman, K., Ahmad, M., 2015. Factors determining energy consumption: Evidence from Indonesia, Malaysia and Thailand. Renew. Sust. Energ. Rev. 42, 1123-1131.

Bailey, D., Katz, J.N., 2011. Implementing Panel Corrected Standard Errors in R: The pcse Package. Journal of Statistical Software 42, 1-11.

Berdiev, A.N., Saunoris, J.W., 2016. Financial development and the shadow economy: A panel VAR analysis. Econ Model 57, 197-207.

Bhattacharyya, S., Hodler, R., 2014. Do Natural Resource Revenues Hinder Financial Development? The Role of Political Institutions. World Dev. 57, 101-113.

Blackburn, K., Bose, N., Capasso, S., 2012. Tax evasion, the underground economy and financial development. Journal of Economic Behavior \& Organization 83, 243-253.

Blundell, R., Bond, S., 1998. Initial conditions and moment restrictions in dynamic panel data models. J. Econom. 87, 115-143.

Botev, J., Égert, B., Jawadi, F., 2019. The nonlinear relationship between economic growth and financial development: Evidence from developing, emerging and advanced economies. Int. Econ. 160, -13.

Canh, N.P., Thanh, S.D., Schinckus, C., Bensemann, J., Thanh, L.T., 2019. Global Emissions: A New Contribution from the Shadow Economy. International Journal of Energy Economics and Policy 9, 320-337. Cavalcante, R.T., Sobreiro, V.A., Kimura, H., 2018. Determinants of the non-life insurance market in Brazil. Rev. Dev. Finance 8, 89-95.

Chen, Z., Huang, W., Zheng, X., 2019. The decline in energy intensity: Does financial development matter? Energy Policy 134, 110945.

Çoban, S., Topcu, M., 2013. The nexus between financial development and energy consumption in the EU: A dynamic panel data analysis. Energy Econ. 39, 81-88.

Dietz, T., Rosa, E.A., 1997. Effects of population and affluence on $\mathrm{CO} 2$ emissions. Proceedings of the National Academy of Sciences 94, 175-179.

Ehrlich, P.R., Holdren, J.P., 1971. Impact of population growth. Science 171, 1212-1217.

Farhani, S., Ozturk, I., 2015. Causal relationship between CO 2 emissions, real GDP, energy consumption, financial development, trade openness, and urbanization in Tunisia. Environ. Sci. Pollut. Res. 22, 1566315676. 
Gaies, B., Kaabia, O., Ayadi, R., Guesmi, K., Abid, I., 2019. Financial development and energy consumption: Is the MENA region different? Energy Policy 135, 111000.

Geng, W., Ming, Z., Lilin, P., Ximei, L., Bo, L., Jinhui, D., 2016. China's new energy development: Status, constraints and reforms. Renew. Sust. Energ. Rev. 53, 885-896.

Hanif, I., 2018. Impact of fossil fuels energy consumption, energy policies, and urban sprawl on carbon emissions in East Asia and the Pacific: A panel investigation. Energy Strateg Rev. 21, 16-24.

Hansen, L.P., 1982. Large sample properties of generalized method of moments estimators. Econometrica: Journal of the Econometric Society, 1029-1054.

Ji, Q., Zhang, D., 2019. How much does financial development contribute to renewable energy growth and upgrading of energy structure in China? Energy Policy 128, 114-124.

Khodzhimatov, R., 2018. XTCOINTREG: Stata module for panel data generalization of cointegration regression using fully modified ordinary least squares, dynamic ordinary least squares, and canonical correlation regression methods.

Le, H.P., Ozturk, I., 2020. The impacts of globalization, financial development, government expenditures, and institutional quality on CO 2 emissions in the presence of environmental Kuznets curve. Environ. Sci. Pollut. Res., 1-18.

Le, T.-H., Nguyen, C.P., Park, D., 2020. Financing renewable energy development: Insights from 55 countries. Energy Research \& Social Science 68, 101537.

Liao, H., Cao, H.-S., 2013. How does carbon dioxide emission change with the economic development? Statistical experiences from 132 countries. Global Environmental Change 23, 1073-1082.

Lin, B., Zhu, J., 2017. Energy and carbon intensity in China during the urbanization and industrialization process: A panel VAR approach. J. Clean. Prod. 168, 780-790.

Maskus, K.E., Milani, S., Neumann, R., 2019. The impact of patent protection and financial development on industrial R\&D. Res. Policy 48, 355-370.

Nasir, M.A., Huynh, T.L.D., Tram, H.T.X., 2019. Role of financial development, economic growth \& foreign direct investment in driving climate change: A case of emerging ASEAN. J. Environ Manage. 242, 131-141. Nguyen, C.P., Nguyen, N.A., Schinckus, C., Su, T.D., 2018. The Ambivalent Role of Institutions in the CO2 Emissions: The Case of Emerging Countries. International Journal of Energy Economics and Policy 8, 7-17. Nickell, S., 1981. Biases in dynamic models with fixed effects. Econometrica: Journal of the Econometric Society, 1417-1426.

Omri, A., Nguyen, D.K., 2014. On the determinants of renewable energy consumption: International evidence. Energy 72, 554-560.

Pan, X., Uddin, M.K., Han, C., Pan, X., 2019a. Dynamics of financial development, trade openness, technological innovation and energy intensity: Evidence from Bangladesh. Energy 171, 456-464.

Pan, X., Uddin, M.K., Saima, U., Guo, S., Guo, R., 2019b. Regime switching effect of financial development on energy intensity: Evidence from Markov-switching vector error correction model. Energy Policy 135, 110995.

Pham, N.M., Huynh, T.L.D., Nasir, M.A., 2020. Environmental consequences of population, affluence and technological progress for European countries: A Malthusian view. J. Environ. Manage. 260, 110143.

Phuc Nguyen, C., Schinckus, C., Dinh Su, T., 2019. Economic integration and CO2 emissions: evidence from emerging economies. Clim. Dev., 1-16.

Pradhan, R.P., Arvin, M.B., Nair, M., Bennett, S.E., Hall, J.H., 2018. The dynamics between energy consumption patterns, financial sector development and economic growth in Financial Action Task Force (FATF) countries. Energy 159, 42-53.

Roodman, D., 2006. How to do xtabond2: An introduction to difference and system GMM in Stata. Center for Global Development Working Paper No. 103.

Roodman, D., 2009. How to do xtabond2: An introduction to difference and system GMM in Stata. The Stata J. 9, 86-136. 
Sadorsky, P., 2013. Do urbanization and industrialization affect energy intensity in developing countries? Energy Econ. 37, 52-59.

Shahbaz, M., Haouas, I., Sohag, K., Ozturk, I., 2020. The financial development-environmental degradation nexus in the United Arab Emirates: the importance of growth, globalization and structural breaks. Environ. Sci. Pollut. Res. 27, 10685-10699.

Shahbaz, M., Nasir, M.A., Roubaud, D., 2018. Environmental degradation in France: The effects of FDI, financial development, and energy innovations. Energy Econ. 74, 843-857.

Svirydzenka, K., 2016. Introducing a new broad-based index of financial development. International Monetary Fund.

Ullah, S., Akhtar, P., Zaefarian, G., 2018. Dealing with endogeneity bias: The generalized method of moments (GMM) for panel data. Ind. Mark. Manag. 71, 69-78.

Ullah, S., Zaefarian, G., Ullah, F., 2020. How to use instrumental variables in addressing endogeneity? A step-by-step procedure for non-specialists. Ind. Mark. Manag.

UN, 2019. About the Sustainable Development Goals. United Nations.

Yang, F., 2019. The impact of financial development on economic growth in middle-income countries. J. Int. Financial Mark 59, 74-89.

Zhang, N., Yu, K., Chen, Z., 2017. How does urbanization affect carbon dioxide emissions? A cross-country panel data analysis. Energy Policy 107, 678-687. 